\title{
Energy Efficient and Robust Balancing with Motion Primitive Switching
}

\author{
Sotiris Apostolopoulos ${ }^{*,+, *}$, Marion Leibold ${ }^{*, \S}$ and Martin Buss, ${ }^{* \dagger,}, \boldsymbol{q}$ \\ *Chair of Automatic Control Engineering, \\ Technical University of Munich, \\ Theresienstraße 90, Munich 80333, Germany \\ ${ }^{\dagger}$ Institute for Advanced Study, \\ Technical University of Munich, \\ Lichtenbergstraße 2a, Garching 85748, Germany \\ \$sotiris.apostolopoulos@tum.de \\ $\S_{\text {marion.leibold@tum.de }}$ \\ ฯmb@tum.de
}

Received 5 August 2016

Accepted 30 January 2017

Published 21 March 2017

\begin{abstract}
Balancing motions are usually designed using simplified models of the Center of Mass (CoM) and feedback control without accounting for energy efficiency. In order to tackle this shortcoming, we introduce a Motion Primitive switching methodology where samples of optimal motions (Motion Primitives) are chosen online based on a Euclidean distance metric. The chosen sample is used to provide reference trajectories, torques and ground reaction forces to be tracked. In order to satisfy all of the modeling assumptions while tracking the reference values, a Quadratic Program (QP) is solved online where the dynamics of the robot, friction, Center of Pressure and torque bounds are treated as constraints. Convergence to the desired trajectories is dictated by a Control Lyapunov Function constraint which is introduced in the QP. The methodology is evaluated on a four-link simulated robot where we show that switching between Motion Primitives provides energy efficient balancing motions for different disturbance situations. At the same time the methodology provides more efficient motions for different disturbance forces when compared to a nonswitching approach, where a Motion Primitive is chosen only once at the beginning.
\end{abstract}

Keywords: Humanoid balancing; Motion Primitives; online optimization.

\section{Introduction}

Humanoid robots have received special attention in the last years due to their versatility and dexterity and a lot of effort is targeted towards endowing them with capabilities for outdoors operation and human assistance. In both cases, the

$\$$ Corresponding author.

This is an Open Access article published by World Scientific Publishing Company. It is distributed under the terms of the Creative Commons Attribution 4.0 (CC-BY) License. Further distribution of this work is permitted, provided the original work is properly cited. 
humanoid should be able to retain its balance, i.e., be able to reject or handle external disturbances such that a fall is avoided. An unexpected disturbance force to a robot should not be considered a rare case, since an only partially observed environment can lead to collisions. In such a case, a control input has to be computed online in order to balance the robot. Also, in assistive tasks like human-robot collaborative object transportation, the robot should be able to counteract or comply with the forces applied by the human partner. In order to reduce the effort from the humanside the appropriate control inputs have to be computed online.

Both cases demonstrate the need for fast computations online. One way to do so is through the utilization of simplified models, like the Linear Inverted Pendulum (LIP) or variations of it, which assume that the mass of the system is concentrated on the Center of Mass (CoM) and is constrained to move at constant height. ${ }^{1,2}$ In that case, the dynamics become linear and the derivation of a control input is simplified. Using such models, different balancing strategies can be achieved like the ankle, the hip, the squat or the stepping strategy. ${ }^{3,4}$ Also, since the dynamics are linear online optimization approaches can be utilized to derive balancing and walking motions and to choose between different balancing strategies. ${ }^{5-7}$

Unfortunately, designing balancing motions using these simplified models does not consider energy consumption since an online optimization using the nonlinear equations of motion of a humanoid is computationally intractable. However, it has been shown that energy consumption is part of the optimization process utilized by humans when performing balancing motions. ${ }^{8}$ Another limitation of using simplified models is that even though the LIP with Reaction Wheel model ${ }^{2}$ has been frequently used in studies of humanoid balancing, the direct application of the resulting motion to the humanoid itself is not straightforward.

Approaches towards the utilization of nonlinear systems of equations suggest the linearization of the system equations around the state of the robot and the use of an LQR controller in the vicinity of the state. For this approach to be used, an estimation of the area of validity of the linearization of the system is necessary. Additionally, the domain of attraction of this LQR controller has to be computed using optimization methods. ${ }^{9}$ Following this process offline for different state instances, the whole state space of the robot can be partitioned in disjoint areas based on the validity of the linearization and the domain of attraction of the corresponding LQR controller leading to the form of an LQR tree. ${ }^{10}$ Humanoid robots however are subject to unilateral constraints on the contact forces, like the friction cone and the Center of Pressure (CoP) constraint, ${ }^{11}$ which are difficult to be taken into account in the LQR tree approach. If these constraints are violated, the modeling assumptions are not valid any more and slipping or underactuation might occur.

A possible solution is to use a database of precomputed trajectories (and/or controllers), so-called Motion Primitives, that satisfy all the modeling assumptions. These Motion Primitives are the outcome of an offline optimization process which takes into account task objectives as well as energy criteria. Each Motion Primitive corresponds to a trajectory/controller that balances the robot with respect to a 
specific disturbance force. For the online utilization, it is then only necessary to properly select the most suitable primitive. Related work regarding balancing with a database of Motion Primitives has been done by Liu and Atkeson where local policies around trajectories are extracted using Differential Dynamic Programming. ${ }^{12}$ In their work however, information of the push magnitude and application point is required for the selection of the local model.

An important aspect when utilizing Motion Primitives is robustness to a wide range of new disturbances, i.e., the ability to balance the robot with respect to disturbances that were not considered during the extraction of the database of the Motion Primitives. For that, online approaches can be utilized to correct any mismatch between the reference values and the measured ones. An example is the Dynamic Balance Force Control ${ }^{13}$ where valid Ground Reaction Forces (GRFs) are either hand tuned or found through the solution of a Quadratic Program (QP). As a next step, dynamically feasible accelerations and torques are generated with the use of a weighted pseudo-inverse. Another approach using pseudo-inversion for animated characters is the Dynamics Filter ${ }^{14}$ and the approach for full body passivity-based control as proposed in the work of Hyon et al. ${ }^{15}$ As an alternative, in the work of Lee and Goswami the balancing motion is generated by solving many QPs sequentially while at the same time desired accelerations are taken into account. ${ }^{16}$ An additional methodology that employs QPs in order to provide motions which satisfy multiple tasks can been found in the work of Herzog et al., where the solution of the proposed QP sequence can achieve rates of $1 \mathrm{kHz} .{ }^{17,18}$ Worth mentioning is also the work of Escande et al., where the solution of a hierarchical QP can provide control rates of $1 \mathrm{kHz}$ as well. ${ }^{19} \mathrm{In}$ addition, Kuindersma et al. solved a QP in order to apply a motion generated by a LIP model to the ATLAS humanoid in a simulated environment. ${ }^{20}$ In the same fashion, in the recent work of Hopkins et al. a QP was used to render the motion generated using the Divergent Component of Motion dynamically feasible and consistent. ${ }^{21,22}$ Finally, reference solutions to different task objectives can also be found using Machine Learning techniques following the approach proposed in our previous work. ${ }^{23}$ In this previous work however, we focused solely on the feasibility of the task and not in preservation of optimality.

In this work, we introduce Motion Primitives in order to provide reference balancing motions. For that, we apply numeric optimization using the nonlinear equations of motion of the robot, while at the same time we take both the CoP and the friction cone constraints into account. For this paper, we adopt a model with four links which is a minimalistic approach of a humanoid robot employing its upper body for angular momentum regulation. Such a model can demonstrate all the balancing strategies, i.e., ankle, hip and squat strategy, but not stepping. In order to create Motion Primitives for different balancing situations, we apply pushes on the middle of the torso with different push strengths. Afterwards, equidistant samples of each trajectory with their corresponding accelerations, torques and GRFs are stored, leading in a database of Motion Primitive Samples. During balancing we apply a Euclidean distance metric on the state to choose the best sample, forming in the end 
a Motion Primitive switching methodology. By using only the state of the robot, we gain also the advantage that we do not depend on information about the size and strength of the push. Otherwise we would need to implement an estimator like the Extended Kalman Filter, such that information regarding the strength and the size of the push could be provided. ${ }^{12}$ We also do not need to apply intensive pre-processing of the data, as was done in the work of Liu and Atkeson. ${ }^{12}$ The reason for that is that due to the lack of any online optimization, a global controller has to be designed such that any control signal applied to the robot can ensure that the modeling assumptions on the GRFs are satisfied. In our QP formulation, such an issue is not the case, since we can online provide balancing motions that satisfy these assumptions. Finally, designing a global controller scales badly with the size of the state of the system in comparison to a static QP.

The robustness to disturbance scenarios not considered during the design of the Motion Primitives is achieved by solving a single QP online where the adopted cost function penalizes deviations from the nominal accelerations, torques and GRFs that are provided by the chosen Motion Primitive. At the same time, torque limits and constraints on the GRFs (friction cone and CoP) are treated as inequality constraints and a Control Lyapunov Function (CLF) constraint is used in order to provide exponential convergence to the desired trajectories at a pre-specified rate. ${ }^{24}$ In that way, the solution of the QP provides an optimal compromise between the tracking of optimized trajectories, the dynamic feasibility of the motion and the satisfaction of the modeling assumptions. We adopted a QP approach instead of a pseudo-inverse one due to the fact that we need to include a CLF constraint. In addition, even though a pseudo-inverse approach can yield solutions that respect the system dynamics, the resulting torques might violate the assumed actuator limits.

The main contribution of our work lies in the switching between pre-computed Motion Primitives in order to provide reference balancing solutions to the robot that bring it back to the rest posture while at the same time they provide robustness to unknown disturbance forces and energy efficient motions. The Motion Primitive switching is proposed, since the solution of the QP might lead the robot state closer to the state of a sample that corresponds to another Motion Primitive. An important characteristic of our work that allows us to monitor the tracking performance is the use of a CLF constraint in the QP. By applying such a switching mechanism, we were able to have a more efficient motion than by committing to a single Motion Primitive from the beginning, something which in our opinion is a finding of major interest.

The remainder of the paper is structured as follows: Sec. 2 presents the dynamics of the four-link robot. In Sec. 3, balancing is formulated as a numeric optimization problem and we explain the procedure for creating the balancing motions and then the database of Motion Primitive Samples. In Sec. 4, we demonstrate how a CLF constraint can be used for tracking. Section 5 presents the QP that we solve online in order to ensure the satisfaction of the constraints that will provide a dynamically feasible and convergent motion, as well as the selection methodology. The simulation results are illustrated and discussed in Sec. 6. Section 7 concludes the paper. 


\section{Dynamic Modeling}

In this section, we introduce the dynamics of the four-link humanoid in the sagittal plane (Fig. 1) which we are going to use as an example to demonstrate our methodology. Please note that the approach is more general and can be applied to 3D models as well. The dynamics are based on the Lagrangian formulation and the assumption of rigid bodies. For the remaining of the paper, the time instance $t$ is omitted as long as it is clear from the context.

\subsection{Model of the four-link humanoid}

The robot has $n=6$ Degrees of Freedom (DoFs) consisting of the joint positions $\boldsymbol{q} \in \mathbb{R}^{n-3}$ as well as the position of the tip and the orientation of the foot $\boldsymbol{p}=\left[\begin{array}{lll}p_{x} & p_{y} & \phi_{z}\end{array}\right]^{T}$. The foot should remain flat on the surface, otherwise underactuation occurs which is an undesired phenomenon. In addition, slipping should be avoided. Assuming $\boldsymbol{\theta}=\left[\begin{array}{ll}\boldsymbol{q}^{T} & \boldsymbol{p}^{T}\end{array}\right]^{T} \in \mathbb{R}^{n}$ and utilizing Lagrangian dynamics, the equations of motion and the flat and pinned foot constraint can be expressed as:

$$
\begin{aligned}
\boldsymbol{D}(\boldsymbol{\theta}) \ddot{\boldsymbol{\theta}}+\boldsymbol{C}(\boldsymbol{\theta}, \dot{\boldsymbol{\theta}}) \dot{\boldsymbol{\theta}}+\boldsymbol{G}(\boldsymbol{\theta}) & =\boldsymbol{S u}+\boldsymbol{J}^{T}(\boldsymbol{\theta}) \boldsymbol{F}, \\
\boldsymbol{J}(\boldsymbol{\theta}) \ddot{\boldsymbol{\theta}}+\dot{\boldsymbol{J}}(\boldsymbol{\theta}) \dot{\boldsymbol{\theta}} & =\mathbf{0}
\end{aligned}
$$

where $\boldsymbol{D}(\boldsymbol{\theta}) \in \mathbb{R}^{n \times n}$ is the mass-inertia matrix, $\boldsymbol{C}(\boldsymbol{\theta}, \dot{\boldsymbol{\theta}}) \in \mathbb{R}^{n \times n}$ is the matrix of centrifugal and Coriolis terms, $\boldsymbol{G}(\boldsymbol{\theta}) \in \mathbb{R}^{n}$ summarizes the gravitational terms, $\boldsymbol{u} \in \mathbb{R}^{m}$ is the vector of generalized torques and $\boldsymbol{S} \in \mathbb{R}^{n \times m}$ is the input matrix. For the four-link humanoid, $m=3$. Finally, $\boldsymbol{F}=\left[\begin{array}{lll}F_{x} & F_{y} & M_{z}\end{array}\right]^{T}$ is the vector of GRFs

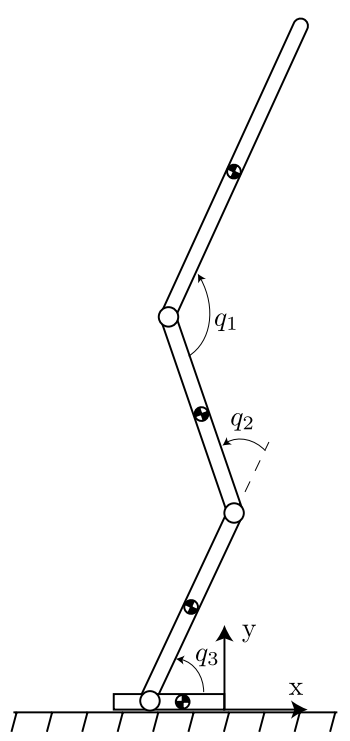

Fig. 1. Model of the planar humanoid used for balancing. The Newtonian reference frame is assumed to be fixed on the ground at the tip of the foot. 
Table 1. Robot parameters.

\begin{tabular}{lcccc}
\hline & Foot & Tibia & Femur & Torso \\
\hline Mass $(\mathrm{kg})$ & 0.4 & 6.4 & 13.6 & 12 \\
Length $(\mathrm{m})$ & 0.2 & 0.4 & 0.4 & 0.63 \\
Inertia $\left(\mathrm{kg} \cdot \mathrm{m}^{2}\right)$ & 0.1 & 0.4 & 0.94 & 1.33 \\
Mass center $(\mathrm{m})$ & 0.12 & 0.16 & 0.29 & 0.24 \\
& (from tip) & (from ankle) & (from knee) & (from hip) \\
\hline
\end{tabular}

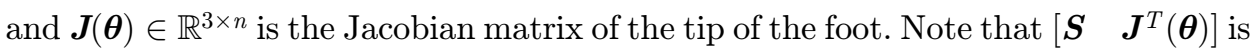
the identity matrix and the right-hand side of Eq. (1a) can be substituted by the concatenation of the input torques $\boldsymbol{u}$ and GRFs $\boldsymbol{F}$, i.e., $\left[\begin{array}{ll}\boldsymbol{u}^{T} & \boldsymbol{F}^{T}\end{array}\right]^{T}$.

The kinematic and dynamic parameters of the robot are presented in Table 1 . The equations of motion were generated using the software AUTOLEV. ${ }^{25}$

\subsection{Rigid impact}

Disturbances to the robot are assumed to take the form of rigid impacts on different points of the torso. An impact is therefore instantaneous and causes a discontinuity in the velocities $\dot{\boldsymbol{\theta}}$, while the positions $\boldsymbol{\theta}$ are left unchanged. Formally, this can be expressed as

$$
\begin{gathered}
\boldsymbol{\theta}^{+}=\boldsymbol{\theta}^{-} \\
\dot{\boldsymbol{\theta}}^{+}=\dot{\boldsymbol{\theta}}^{-}+\boldsymbol{D}^{-1}\left(\boldsymbol{\theta}^{-}\right) \boldsymbol{J}_{\mathrm{push}}^{T}\left(\boldsymbol{\theta}^{-}\right) \boldsymbol{F}_{\mathrm{push}},
\end{gathered}
$$

where the plus and minus superscripts denote the post-impact and pre-impact state of the system, respectively. The matrix $\boldsymbol{J}_{\text {push }}(\boldsymbol{\theta}) \in \mathbb{R}^{2 \times n}$ denotes the Jacobian of the impact point and the vector $\boldsymbol{F}_{\text {push }} \in \mathbb{R}^{2}$ denotes the impact force applied to the robot.

\section{Motion Primitives for Humanoid Balancing}

In this section, we describe the static optimization problem that leads to the design of the Motion Primitives. Afterwards, we explain how each primitive is sampled and stored in the memory in order to form a database of Motion Primitive Samples.

\subsection{Balancing as an optimization problem}

For this work we choose to formulate the balancing problem as a trajectory optimization problem. We parametrize each joint trajectory as a Bézier polynomial, i.e.,

$$
q_{i}(t)=\sum_{k=0}^{M} \alpha_{k}^{i} \frac{M !}{k !(M-k) !}\left(\frac{t}{T}\right)^{k}\left(\frac{T-t}{T}\right)^{M-k},
$$

where $M$ is the order and $\alpha_{k}^{i}$ the coefficients of the Bézier polynomial. The total duration of the balancing motion $T$ is fixed. We do not include in the trajectory optimization the position of the tip and the orientation of the foot contained in $\boldsymbol{p}$, 
since they have to be constantly zero in order to satisfy Eq. (1b), i.e., the contact constraint.

The cost function to be minimized is adopted by the work from Atkeson and Stephens ${ }^{3}$ and is given by

$$
I(\boldsymbol{\alpha})=\int_{0}^{T}\left\{\left(\boldsymbol{q}-\boldsymbol{q}_{0}\right)^{T} \boldsymbol{W}_{1}\left(\boldsymbol{q}-\boldsymbol{q}_{0}\right)+\dot{\boldsymbol{q}}^{T} \boldsymbol{W}_{2} \dot{\boldsymbol{q}}+\boldsymbol{u}^{T} \boldsymbol{W}_{3} \boldsymbol{u}\right\} \mathrm{dt},
$$

where $\boldsymbol{\alpha}=\left[\boldsymbol{\alpha}^{1} \cdots \boldsymbol{\alpha}^{n}\right] \in \mathbb{R}^{(M+1) \times n}$ are the Bézier coefficients of each joint trajectory and $\boldsymbol{W}_{i}$ are weighting matrices of appropriate dimensions. Note that $\boldsymbol{q}, \dot{\boldsymbol{q}}$ and $\boldsymbol{u}$ depend on $\boldsymbol{\alpha}$ but in the definition this notation is suppressed for brevity. Finally, $\boldsymbol{q}_{0}$ corresponds to the desired rest posture of the robot. The cost function minimizes deviations from the rest posture, while at the same time it keeps the joint torques relatively low, such that energy efficiency is also taken into account. The weighting between these two objectives is determined by the matrices $\boldsymbol{W}_{i}$. It should be noted that this cost function can provide motions that use the ankle, hip and squat strategies. In addition, one can include the time $T$ in the optimization variables in an effort to compute more efficient balancing motions for larger push sizes, but an increase in complexity and convergence time should be expected.

During the optimization the following constraints have to be imposed such that the modeling assumptions are satisfied, foot tilting is avoided as well as actuator and joint limits are satisfied.

- Center of Pressure (CoP) constraint: The $\mathrm{CoP}^{11}$ is defined as the point where the total sum of contact forces acts and causes a force but not a moment. Formally it is defined as

$$
\mathrm{CoP}=\frac{M_{z}}{F_{y}},
$$

where $M_{z}$ is the ground reaction moment and $F_{y}$ the vertical GRF. As long as the $\mathrm{CoP}$ is inside the base of support, foot tilting is avoided and the CoP is equivalent to the Zero Moment Point. ${ }^{26}$ With correspondence to Fig. 1 the CoP constraint can be stated as

$$
-\ell_{\text {foot }} \leq \mathrm{CoP} \leq 0
$$

where $\ell_{\text {foot }}$ is the length of the foot, equal to $0.2 \mathrm{~m}$.

- Friction cone constraint: In order to ensure that the foot is not sliding, the following relation must be satisfied:

$$
-\mu_{s} F_{y} \leq F_{x} \leq \mu_{s} F_{y}
$$

where $\mu_{s} \in \mathbb{R}$ is the coefficient of static friction.

- Positive vertical contact force:

$$
F_{y}>0
$$


- Joint position and joint velocity limits:

$$
\begin{aligned}
& \boldsymbol{q}_{\min } \leq \boldsymbol{q} \leq \boldsymbol{q}_{\max }, \\
& \dot{\boldsymbol{q}}_{\min } \leq \dot{\boldsymbol{q}} \leq \dot{\boldsymbol{q}}_{\max }
\end{aligned}
$$

- Input torque saturation:

$$
\boldsymbol{u}_{\min } \leq \boldsymbol{u} \leq \boldsymbol{u}_{\max }
$$

This optimization process is directly applicable to fully actuated robots. The advantage of using parametrized trajectories is that the accelerations $\ddot{\boldsymbol{q}}$, control inputs $\boldsymbol{u}$ and GRFs $\boldsymbol{F}$ have analytical expressions. On the other hand, Bézier polynomials have the advantage that the first two coefficients $\boldsymbol{a}_{0}^{i}$ and $\boldsymbol{a}_{1}^{i}$ of each DoF $i$ are determined by the initial state of the robot and the last two coefficients $\boldsymbol{a}_{M-1}^{i}$ and $\boldsymbol{a}_{M}^{i}$ are determined by the terminal state of the robot. Another advantage of the Bézier parametrization is that the control inputs $\boldsymbol{u}$ are smooth in contrast to the control inputs returned from an optimal control algorithm, which are in principle piecewise linear or piecewise constant. Of course, one can assume that the control inputs are states and the new control inputs are the torque derivatives. As a consequence though, the dimensionality of both the system and the optimization problem is increased and convergence is more difficult to be achieved. More insight on how optimal control could have been used to generate motions for humanoids can be found in the work of Denk and Schmidt ${ }^{27}$ and Koch et al. ${ }^{28}$

Alternatives include Machine Learning approaches like Reinforcement Learning $^{29-31}$ or the use of Dynamical Movement Primitives (DMPs) which have been widely used for robotic applications. ${ }^{32-34}$ In comparison to the Beziér parametrization, they are described by more coefficients (kernel weights). In lack of demonstration data, these weights can be learned by Reinforcement Learning approaches, which in principle require more computational time. ${ }^{35}$ However, the DMPs are in general characterized by better scaling properties both spatial and temporal.

\subsection{Database of motion primitives samples}

In order to generate the database of Motion Primitives, we apply impact forces on the middle of the torso and use (2) to find the initial state of the robot. Then we solve the aforementioned static optimization problem (Sec. 3.1) using the fmincon function in MATLAB and a database of balancing motions (Motion Primitives) is generated.

As a next step, each Motion Primitive is sampled every $T_{s}$ seconds. For each Motion Primitive the important quantities that are stored in the database of Motion Primitive samples are the joint positions, velocities and accelerations, as well as the control inputs and GRFs at each time step $k T_{s}, k=0, \ldots, \frac{T}{T_{s}}$. The time step $k T_{s}$ that corresponds to the sample is also needed as well as the index $j$ of the current Motion Primitive. The Motion Primitive with index $j$ will be used to generate the reference values until we choose a new sample or until the end of the balancing motion in the 
case where the switching is shut off, as no other primitive is used. Summarizing, a Motion Primitive sample is formally defined as the tuple

$$
P_{[j, k]}=\left(\boldsymbol{\theta}\left(k T_{s}\right), \dot{\boldsymbol{\theta}}\left(k T_{s}\right), \ddot{\boldsymbol{\theta}}\left(k T_{s}\right), \boldsymbol{u}\left(k T_{s}\right), \boldsymbol{F}\left(k T_{s}\right)\right) .
$$

Therefore, the database $\mathcal{D}$ of Motion Primitives samples is defined as the collection $\mathcal{D}=\bigcup_{j} \bigcup_{k} P_{[j, k]}$. By keeping the index $j$ constant and iterating through all the samples with $k$ values from $k=0$ to $k=\frac{T}{T_{s}}$ we can reproduce a Motion Primitive.

Please note that as an alternative we could store only the Bézier coefficients $\boldsymbol{\alpha}$ and generate the reference trajectories online. Then, by computing the system matrices we can compute the input torques and the GRFs. This is a trade-off between memory and CPU power. In our work we do not want to introduce any computational overhead and as a consequence we avoid storing only the Bézier coefficients $\boldsymbol{\alpha}$.

\section{CLF for Trajectory Tracking}

In this section, we briefly introduce the concept of the CLF which is utilized to provide feedback policies such that the desired trajectories will be tracked exponentially. ${ }^{36,37}$ The CLF is utilized because we do not want to explicitly determine any feedback gain matrices, but allow them to be decided online. The reason for that is that predetermining the gains might lead to undesired behavior. Low gains can lead to poor tracking, while large gains can lead to large accelerations that as a consequence can induce GRFs that violate the modeling assumptions or can produce large torques that exceed the actuator constraints.

\subsection{Motion primitive tracking}

For trajectory tracking we define outputs $\boldsymbol{y}$ of the form

$$
\boldsymbol{y}=\boldsymbol{\theta}-\boldsymbol{\theta}_{d},
$$

where $\boldsymbol{\theta}_{d}$ are the desired trajectories that are designed using the methodology that was described in Sec. 3.1.

In order to zero the outputs we choose the Computed Torque Control (CTC) ${ }^{38}$ scheme which is based on Input-Output Feedback Linearization. In CTC the control input is defined as

$$
\left[\begin{array}{c}
\boldsymbol{u} \\
\boldsymbol{F}
\end{array}\right]=\boldsymbol{D}\left(\ddot{\boldsymbol{\theta}}_{d}+\frac{1}{\varepsilon} \boldsymbol{K}_{D}\left(\dot{\boldsymbol{\theta}}_{d}-\dot{\boldsymbol{\theta}}\right)+\frac{1}{\varepsilon^{2}} \boldsymbol{K}_{P}\left(\boldsymbol{\theta}_{d}-\boldsymbol{\theta}\right)\right)+\boldsymbol{C}+\boldsymbol{G},
$$

where $\boldsymbol{K}_{D}$ and $\boldsymbol{K}_{P}$ are positive definite constant diagonal matrices (gain matrices). Then, we define:

$$
\begin{aligned}
& \ddot{\boldsymbol{\theta}}=\ddot{\boldsymbol{\theta}}_{d}+\frac{1}{\varepsilon} \boldsymbol{K}_{D}\left(\dot{\boldsymbol{\theta}}_{d}-\dot{\boldsymbol{\theta}}\right)+\frac{1}{\varepsilon^{2}} \boldsymbol{K}_{P}\left(\boldsymbol{\theta}_{d}-\boldsymbol{\theta}\right), \\
& \ddot{\boldsymbol{\theta}}=\ddot{\boldsymbol{\theta}}_{d}+\boldsymbol{w}
\end{aligned}
$$


and then the closed loop dynamics become

$$
\begin{aligned}
{\left[\begin{array}{c}
\dot{y} \\
\ddot{y}
\end{array}\right] } & =\left[\begin{array}{ll}
0 & I \\
0 & 0
\end{array}\right]\left[\begin{array}{l}
y \\
\dot{y}
\end{array}\right]+\left[\begin{array}{l}
0 \\
I
\end{array}\right] w \\
& \Rightarrow \dot{\boldsymbol{\eta}}=\boldsymbol{A} \boldsymbol{\eta}+\boldsymbol{B} w .
\end{aligned}
$$

Considering the continuous time algebraic Riccati equation

$$
\boldsymbol{A}^{T} \boldsymbol{P}+\boldsymbol{P} \boldsymbol{A}-\boldsymbol{P} \boldsymbol{B} \boldsymbol{B}^{T} \boldsymbol{P}+\boldsymbol{Q}=\mathbf{0},
$$

where the solution is $\boldsymbol{P}$, we can use the solution $\boldsymbol{P}$ to construct a Rapidly Exponentially Stabilizing (RES)-CLF that can stabilize (15) at a rate $0<\varepsilon<1$. Note that $\boldsymbol{P}$ and $\boldsymbol{Q}$ are positive definite symmetric matrices.

The Lyapunov function $V$ has a quadratic form

$$
V(\boldsymbol{\eta})=\boldsymbol{\eta}^{T}\left[\begin{array}{cc}
\frac{1}{\varepsilon} \boldsymbol{I} & \mathbf{0} \\
\mathbf{0} & \boldsymbol{I}
\end{array}\right] \boldsymbol{P}\left[\begin{array}{cc}
\frac{1}{\varepsilon} \boldsymbol{I} & \mathbf{0} \\
\mathbf{0} & \boldsymbol{I}
\end{array}\right] \boldsymbol{\eta}=\boldsymbol{\eta}^{T} \boldsymbol{P}_{\varepsilon} \boldsymbol{\eta}
$$

and as defined in the work of Ames et $a l .{ }^{24}$ it is a RES-CLF if the following two conditions hold:

- The CLF is bounded as:

$$
c_{1}\|\boldsymbol{\eta}\|^{2} \leq V(\boldsymbol{\eta}) \leq \frac{c_{2}}{\varepsilon^{2}}\|\boldsymbol{\eta}\|^{2},
$$

where $c_{1}>0$ and $c_{2}>0$.

- The CLF is exponentially decreasing:

$$
\begin{aligned}
& \dot{V}(\boldsymbol{\eta}) \leq-\frac{c_{3}}{\varepsilon} V(\boldsymbol{\eta}), \\
& \boldsymbol{\eta}^{T}\left(\boldsymbol{A}^{T} \boldsymbol{P}_{\varepsilon}+\boldsymbol{P}_{\varepsilon} \boldsymbol{A}\right) \boldsymbol{\eta}+2 \boldsymbol{\eta}^{T} \boldsymbol{P}_{\varepsilon} \boldsymbol{B} \boldsymbol{w} \leq-\frac{c_{3}}{\varepsilon} V(\boldsymbol{\eta}), \\
& \boldsymbol{\eta}^{T}\left(\boldsymbol{A}^{T} \boldsymbol{P}_{\varepsilon}+\boldsymbol{P}_{\varepsilon} \boldsymbol{A}\right) \boldsymbol{\eta}+\frac{c_{3}}{\varepsilon} V(\boldsymbol{\eta})+2 \boldsymbol{\eta}^{T} \boldsymbol{P}_{\varepsilon} \boldsymbol{B} \boldsymbol{w} \leq 0, \\
& \psi_{0}+\boldsymbol{\psi}_{1}^{T} \boldsymbol{w} \leq 0,
\end{aligned}
$$

where $c_{3}$ is defined as

$$
c_{3}=\frac{\lambda_{\min }(\boldsymbol{Q})}{\lambda_{\max }(\boldsymbol{P})}>0
$$

and $\lambda_{\min }(\cdot)$ and $\lambda_{\max }(\cdot)$ denote the minimum and maximum eigenvalues, respectively, of a given symmetric matrix.

For the derivation of (19) we made use of (15) and the fact that $\boldsymbol{P}_{\varepsilon}$ is symmetric.

\subsection{Feedback determination through $Q P$ with $C L F$ constraint}

The auxiliary input $\boldsymbol{w}$ can be determined online through the solution of a QP. A simple version of it finds the minimum norm $\boldsymbol{w}$ which satisfies (19) with a tolerance $\delta$ 
and can be formulated as

$$
\begin{aligned}
& \underset{(\delta, \boldsymbol{w})}{\arg \min }\left\{p \delta^{2}+\boldsymbol{w}^{T} \boldsymbol{w}\right\} \\
& \text { s.t. } \quad \psi_{0}+\boldsymbol{\psi}_{1}^{T} \boldsymbol{w} \leq \delta,
\end{aligned}
$$

where $p$ is a positive scalar that penalizes the violation $\delta$ of (19). The CLF constraint is relaxed because otherwise it can lead to a feedback $\boldsymbol{w}$ that in order to be realized needs torques that exceed the actuator limits or GRFs that violate the modeling assumptions. As shown in the work of Ames and Power, this formulation allows for constraints on the torques and GRFs. ${ }^{39}$

\section{Robustness to Unknown Disturbances}

The purpose of using a database of Motion Primitives samples is to be able to provide an energy efficient and dynamically feasible reference balancing motion that satisfies all the modeling assumptions for a wide range of impact situations. At the same time it assists in steering the motion of the robot towards the rest posture, after it was being disturbed with an impact force that was not considered during the generation of the Motion Primitives.

As a consequence, our approach will be able to balance the robot with respect to unknown situations, demonstrating robustness characteristics. For this work, we define as state of the robot the vector $\boldsymbol{x}$ of positions and velocities, i.e., $\boldsymbol{x}=\left[\begin{array}{ll}\boldsymbol{\theta}^{T} & \dot{\boldsymbol{\theta}}^{T}\end{array}\right]^{T}$ and disturbances correspond to impact forces with a magnitude and/or an application point which was not considered during the generation of the Motion Primitives, as described in Sec. 3.2. In principle, robustness is achieved by choosing a suitable Motion Primitive sample, then solving a QP at each time step to ensure that the constraints on the GRFs $\boldsymbol{F}$ and control inputs $\boldsymbol{u}$ which were introduced at the static optimization problem in Sec. 3.1 are satisfied and that the resulting motion is dynamically feasible and stabilizing according to the CLF (17).

\subsection{Selection of new samples - Motion Primitive switching}

The selection methodology takes place every $T_{n}$ seconds, where $T_{n}$ is greater or equal to the sampling time $T_{s}$. This is done because the size of the database and the computational resources available might prevent the ideal case of $T_{n}=T_{s}$. Every $T_{n}$ seconds we apply a weighted Euclidean distance metric on the current state of the robot $\boldsymbol{x}\left(k T_{n}\right)$ and the samples in the database $\mathcal{D}$, i.e.,

$$
P^{*}=\underset{i, k}{\arg \min }\left(P_{[i, k]}^{\boldsymbol{x}}-\boldsymbol{x}\left(k T_{n}\right)\right)^{T} \boldsymbol{L}\left(P_{[i, k]}^{\boldsymbol{x}}-\boldsymbol{x}\left(k T_{n}\right)\right) .
$$

Here, the matrix $\boldsymbol{L}$ is a weighting matrix.

The reason for the switching is due to the fact that the tracking might have poor performance at the expense of satisfying the modeling assumptions or the torque limits. For that reason, it might be preferable to find a better Motion Primitive to track. 


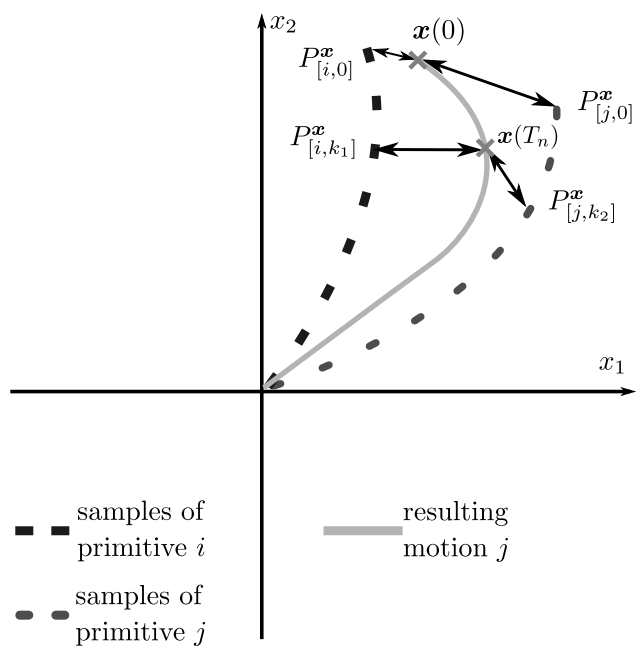

Fig. 2. Graphic representation of the switching approach. The initial state $\boldsymbol{x}(0)$ is closer to the state of the sample $P_{[i, 0]}$, but after $T_{n}$ seconds we find that the trajectory of another Motion Primitive is closer to $\boldsymbol{x}\left(T_{n}\right)$ and for that reason we choose to track the Motion Primitive with index $j$. Afterwards, either the trajectory of the $j$ th-Motion Primitive is always the closest in terms of Euclidean distance or the CLF constraint violation at time $T_{n}$ was below the user defined threshold $\delta_{\text {thr }}$ and we shut off the switching. The dummy states $x_{1}$ and $x_{2}$ are introduced for presentation purposes and $(0,0)$ is the equilibrium state.

If switching is preferable, for the next $T_{n}$ seconds the index $j$ of $P^{*}$ will be used to generate the desired joint positions, velocities, accelerations, torques and GRFs for the QP. The QP is solved at each execution step, i.e., every $T_{s}$ seconds. The switching is turned off at a time $t^{*}$ when the CLF violation $\delta$ is smaller than a user defined threshold $\delta_{\text {thr }}$ and for the remaining $T-t^{*}$ seconds the desired values are generated from the last selected Motion Primitive. In Fig. 2 we show a graphic representation of the switching methodology.

\subsection{QP for Motion Primitive tracking and constraint satisfaction}

The tracking of a Motion Primitive is expressed as a QP, allowing to be solved online. For the QP, we have to define a quadratic cost function and express all the necessary equality and inequality constraints in a linear way. In this work we choose to give more freedom to the optimization problem by allowing it to manipulate not only the violation $\delta$ of the CLF constraint (19), the torques $\boldsymbol{u}$ and the GRFs $\boldsymbol{F}$, but also the accelerations $\ddot{\boldsymbol{q}}$, as opposed to the work of Ames and Powell. ${ }^{39}$ The cost function $\Phi$ to be minimized is defined as

$$
\Phi=\left\{\left(\left[\begin{array}{c}
\ddot{\boldsymbol{\theta}}^{*} \\
0 \\
\boldsymbol{u}^{*} \\
\boldsymbol{F}^{*}
\end{array}\right]-\left[\begin{array}{c}
\ddot{\boldsymbol{\theta}} \\
\delta \\
\boldsymbol{u} \\
\boldsymbol{F}
\end{array}\right]\right) \boldsymbol{H}\left(\left[\begin{array}{c}
\ddot{\boldsymbol{\theta}}^{*} \\
0 \\
\boldsymbol{u}^{*} \\
\boldsymbol{F}^{*}
\end{array}\right]-\left[\begin{array}{c}
\ddot{\boldsymbol{\theta}} \\
\delta \\
\boldsymbol{u} \\
\boldsymbol{F}
\end{array}\right]\right)\right\}
$$


and is penalizing deviations from the reference values provided by the selected primitive which is given by the index $j$ of $P^{*}$. In the function above, $\boldsymbol{H}$ is a positive definite weighting matrix, where the diagonal element $\boldsymbol{H}_{n+1, n+1}$ equals $p$ and controls the penalty $\delta$ on the violation of the CLF constraint (19). Regarding the linear constraints introduced in the QP, they are listed below with a brief description of their purpose.

\subsubsection{Dynamically feasible motion}

The motion that is required to be performed by the robot should be dynamically feasible. In order to ensure this, we express the dynamics of the robot (1) as

$$
\underbrace{\left[\begin{array}{cccc}
\boldsymbol{D} & 0 & -\boldsymbol{S} & -\boldsymbol{J}^{T} \\
\boldsymbol{J} & 0 & \mathbf{0} & \mathbf{0}
\end{array}\right]}_{A_{\mathrm{eq}}}\left[\begin{array}{c}
\ddot{\boldsymbol{\theta}} \\
\delta \\
\boldsymbol{u} \\
\boldsymbol{F}
\end{array}\right]=\underbrace{\left[\begin{array}{c}
-\boldsymbol{C}-\boldsymbol{G} \\
-\dot{\boldsymbol{J}} \boldsymbol{\theta}
\end{array}\right]}_{b_{\mathrm{eq}}}
$$

which will be imposed in the QP as an equality constraint.

\subsubsection{CLF constraint}

The CLF constraint has been already discussed in Sec. 4 and here is rewritten in a form that is suitable for the proposed QP. To that end, Eq. (19) can be brought in the form of

$$
\underbrace{\left[\begin{array}{llll}
\boldsymbol{\psi}_{1}^{T} & -1 & \mathbf{0} & \mathbf{0}
\end{array}\right]}_{A_{\mathrm{in}}^{1}}\left[\begin{array}{c}
\ddot{\boldsymbol{\theta}} \\
\delta \\
\boldsymbol{u} \\
\boldsymbol{F}
\end{array}\right] \leq \underbrace{-\psi_{0}+\boldsymbol{\psi}_{1}^{T} \ddot{\boldsymbol{\theta}}^{*}}_{b_{\mathrm{in}}^{1}}
$$

since $\boldsymbol{w}=\ddot{\boldsymbol{\theta}}-\ddot{\boldsymbol{\theta}}^{*}$.

\subsubsection{Constraints on the contact forces}

The constraints imposed on the contact forces can be expressed as inequality constraints which are linear to the forces. This can be expressed as follows

$$
\underbrace{\left[\begin{array}{cccccc}
\mathbf{0} & 0 & \mathbf{0} & 1 & -\mu_{s} & 0 \\
\mathbf{0} & 0 & \mathbf{0} & -1 & -\mu_{s} & 0 \\
\mathbf{0} & 0 & \mathbf{0} & 0 & -1 & 0 \\
\mathbf{0} & 0 & \mathbf{0} & 0 & 0 & 1 \\
\mathbf{0} & 0 & \mathbf{0} & 0 & -\ell_{\mathrm{foot}} & -1
\end{array}\right]}_{A_{\mathrm{in}}^{2}}\left[\begin{array}{c}
\ddot{\boldsymbol{\theta}} \\
\delta \\
\boldsymbol{u} \\
\boldsymbol{F}
\end{array}\right] \leq \underbrace{\left[\begin{array}{c}
0 \\
0 \\
-\epsilon \\
0 \\
0
\end{array}\right]}_{b_{\mathrm{in}}^{2}}
$$

where $\epsilon$ is a small positive number. The first two lines correspond to the friction cone constraint. The third one ensures that the ground is pushing instead of pulling. The last two lines correspond to the $\mathrm{CoP}$ constraint. The variable $\ell_{\text {foot }}$ corresponds to the length of the foot. 


\subsubsection{Torque limits}

Finally, we impose a linear inequality constraint for the torque limits such that unacceptable joint torques are avoided.

$$
\underbrace{\left[\begin{array}{cccc}
\mathbf{0} & 0 & \boldsymbol{I} & \mathbf{0} \\
\mathbf{0} & 0 & -\boldsymbol{I} & \mathbf{0}
\end{array}\right]}_{A_{\mathrm{in}}^{3}}\left[\begin{array}{c}
\ddot{\boldsymbol{\theta}} \\
\delta \\
\boldsymbol{u} \\
\boldsymbol{F}
\end{array}\right] \leq \underbrace{\left[\begin{array}{r}
\boldsymbol{u}_{\max } \\
-\boldsymbol{u}_{\min }
\end{array}\right]}_{b_{\mathrm{in}}^{3}}
$$

\subsection{Overall $Q P$}

For brevity, the optimization variables can be concatenated in $\boldsymbol{\kappa}=$ $\left[\begin{array}{llll}\ddot{\boldsymbol{q}}^{T} & \delta & \boldsymbol{u}^{T} & \boldsymbol{F}^{T}\end{array}\right]^{T}$ and then the overall QP is formulated as

$$
\begin{aligned}
& \arg \min _{\boldsymbol{\kappa}} \Phi \\
& \text { s.t. } \quad \boldsymbol{A}_{\mathrm{eq}} \boldsymbol{\kappa}=\boldsymbol{b}_{\mathrm{eq}}
\end{aligned}
$$

$$
\left[\begin{array}{c}
\boldsymbol{A}_{\text {in }}^{1} \\
\boldsymbol{A}_{\text {in }}^{2} \\
\boldsymbol{A}_{\text {in }}^{3}
\end{array}\right] \boldsymbol{\kappa} \leq\left[\begin{array}{c}
\boldsymbol{b}_{\text {in }}^{1} \\
\boldsymbol{b}_{\text {in }}^{2} \\
\boldsymbol{b}_{\text {in }}^{3}
\end{array}\right] .
$$

This QP finds a compromise between staying close to the reference acceleration, torques and GRFs provided by the primitive which is given by the chosen sample $P^{*}$ and at the same time keeping the CLF violation $\delta$ close to zero (or optimally zero) such that we achieve good tracking performance. At the same time, we avoid unacceptable torques $\boldsymbol{u}$ and GRFs $\boldsymbol{F}$ that violate the modeling assumptions or accelerations $\ddot{\boldsymbol{\theta}}$ which in combination with the torques $\boldsymbol{u}$ and the forces $\boldsymbol{F}$ will give rise to dynamically infeasible motions. In Fig. 3 we depict the complete architecture of the proposed methodology.

At this point we would like to mention that the QP approach can provide balancing solutions for disturbance scenarios where the offline optimization presented in Sec. 3.1 fails. This is due to the fact that the offline optimization utilizes Bezier polynomials which are smooth and as a consequence provide smooth control inputs $\boldsymbol{u}$ and GRFs $\boldsymbol{F}$. In contrast, the QP provides control inputs and GRFs that do not necessarily have to be fitted by any spline since they are computed in every time step $T_{s}$. As a consequence the QP allows for more arbitrarily-shaped control inputs and GRFs to be generated that in turn can provide balancing motions that are capable of dealing with a wider variety of disturbance scenarios.

One might have been successful in generating balancing solutions for the case where the offline Beziér-based approach fails by using Optimal Control with piecewise constant or linear parametrization for the control inputs. Finally, an enhancement of the Beziér polynomials is possible by increasing their order $M$ or allowing for nonequally-spaced control points, but an increase in complexity is expected. 


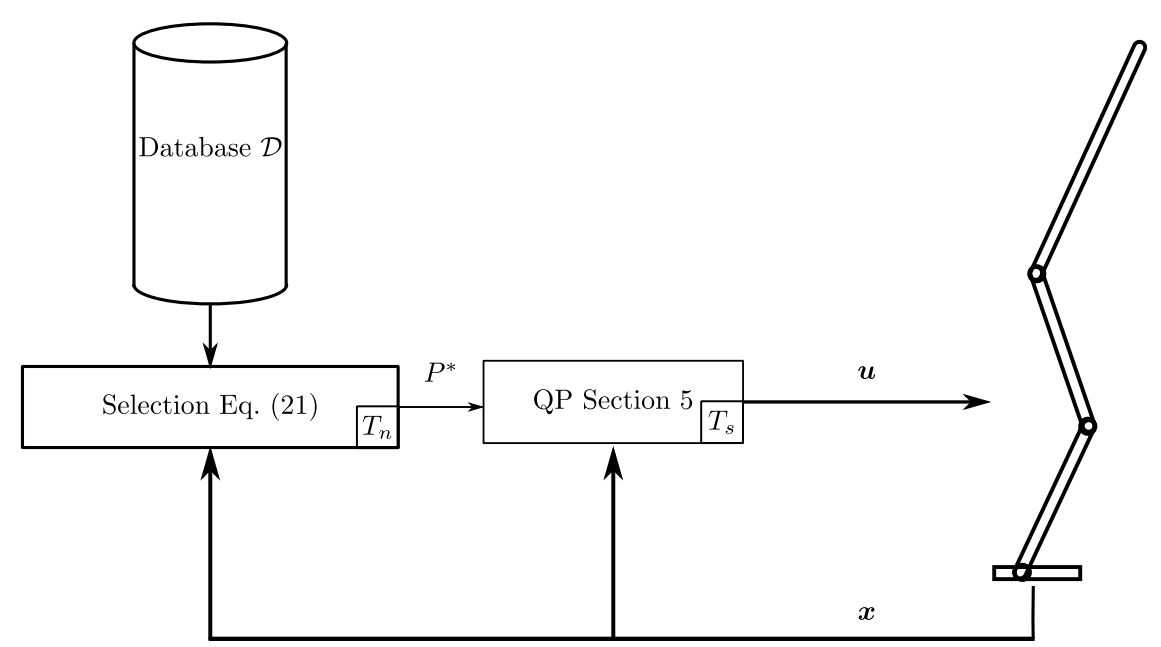

Fig. 3. Schematic diagram of the overall proposed architecture. The working frequency of the QP block is $T_{s}$ seconds while a new sample is selected every $T_{n}$ seconds. The selection block is shut off if the CLF violation $\delta$ is smaller than a threshold $\delta_{\mathrm{thr}}$. If this is the case the index of the last selected sample dictates the Motion Primitive that provides reference values to the QP for the remaining of the balancing motion.

\section{Simulation Evaluation}

This section presents the evaluation of the aforementioned methodology on a simulated four-link robot. We first list the values of the parameters and the variables used in this work in order to facilitate the reproducibility of the results reported here. Later, we show different evaluation scenarios.

\subsection{Database extraction - values and parameters}

The balancing motions are generated by solving the static optimization problem in Sec. 3 for impact forces of the form $\boldsymbol{F}_{\text {push }}=\left[\begin{array}{ll}f & 0\end{array}\right]^{T}$ with $f=1, \ldots, 80 \mathrm{~N}$ with an interval of $1 \mathrm{~N}$. The application point of the impact force is at the middle of the torso. Therefore, we have a total of 80 optimization problems to solve offline. After that, each primitive is sampled every $T_{s}$ seconds leading to a database $\mathcal{D}$ of $\left(\frac{T}{T_{s}}+1\right) \times$ $80=3001 \times 80=240,080$ Motion Primitive samples. Note that in the sequel the application point of an impact force will be described by a number between 0 and 1 , where 0 means the hip and 1 the end-point of the torso. Following that, the application point for the generation of the Motion Primitives was 0.5.

The parameters of the static optimization problem are presented in Table 2 and the ones of the QP and the selection methodology in Table 3. For the solution of the $\mathrm{QP}$ in the online phase we used the open source software qpOASES. ${ }^{40}$

In Table 3, the zero elements of $\boldsymbol{L}$ correspond to the tip position and foot orientation $\boldsymbol{p}$ and the tip velocity and foot angular velocity $\dot{\boldsymbol{p}}$, which are always zero. The nonzero elements correspond to the joint positions $\boldsymbol{q}$ and velocities $\dot{\boldsymbol{q}}$. The rest of the values of the weighting matrix $\boldsymbol{L}$ are experimentally tuned. In principle, however, 
Table 2. Parameters and variables used for the extraction of the Motion Primitives.

\begin{tabular}{|c|c|c|}
\hline Cost function $I$ & $\begin{array}{l}\boldsymbol{W}_{1} \\
\boldsymbol{W}_{2} \\
\boldsymbol{W}_{3}\end{array}$ & $\begin{array}{c}\operatorname{diag}\left(\left[\begin{array}{lll}1 & 1 & 1\end{array}\right]\right) \\
\operatorname{diag}\left(\left[\begin{array}{lll}1 & 1 & 1\end{array}\right]\right) \\
\operatorname{diag}\left(\left[\begin{array}{lll}0.002 & 0.002 & 0.002\end{array}\right]\right)\end{array}$ \\
\hline Total time $(\mathrm{s})$ & $T$ & 3 \\
\hline Order of Bézier polynomial & $M$ & 5 \\
\hline Static friction coefficient & $\mu_{s}$ & 0.7 \\
\hline
\end{tabular}

Table 3. Parameters and variables used in the Motion Primitive sample selection and in the QP.

\begin{tabular}{|c|c|c|}
\hline Selection method & $\begin{array}{c}\boldsymbol{L} \\
T_{s}(\mathrm{~ms}) \\
T_{n}(\mathrm{~ms}) \\
\delta_{\mathrm{thr}}\end{array}$ & $\operatorname{diag}\left(\left[\begin{array}{llllllllllll}1 & 5 & 10 & 0 & 0 & 0 & 0.1 & 0.5 & 1 & 0 & 0 & 0\end{array}\right]\right)$ \\
\hline CLF constraint & $\begin{array}{l}\epsilon \\
p \\
Q\end{array}$ & 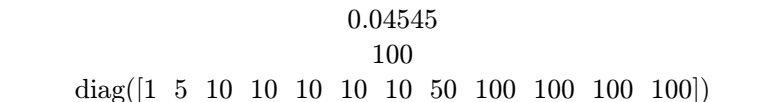 \\
\hline $\begin{array}{l}\text { Cost function } \\
\text { Torque limits }\end{array}$ & $\begin{array}{c}\boldsymbol{H} \\
\boldsymbol{u}_{\max }(\mathrm{N} \cdot \mathrm{m}) \\
\boldsymbol{u}_{\min }(\mathrm{N} \cdot \mathrm{m})\end{array}$ & 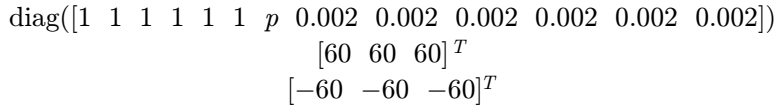 \\
\hline
\end{tabular}

for $\boldsymbol{L}$ and $\boldsymbol{Q}$ we use higher penalties for elements that correspond to DoFs that are closer to the foot. The reason for that is that they have to manipulate the masses and inertia of all the links above them in the kinematic chain.

\subsection{Trajectory switching}

In this experiment we apply an impact force $\boldsymbol{F}_{\text {push }}=\left[\begin{array}{ll}40 & 25\end{array}\right]^{T}$ with an application point of 0.7 . The nominal cost $I$ (see Eq. (4)) of the overall produced motion is 7.1755. For comparison purposes, we also used the offline optimization process described in Sec. 3 for the same impact force. Remarkably, the offline optimization for this disturbance scenario gave a solution with an overall cost of 7.1740 , initialized from different Bézier coefficients randomly. This is a very interesting finding, since the QP solution was able to come up with a balancing motion that is very close to the optimal one, only by switching between optimized balancing motions.

As shown in Fig. 4, the robot returns finally to the rest posture. In addition, the torques (Fig. 5) always stay in the limits and after some transition phase at the beginning, they remain smooth. The constraints on the GRFs (Fig. 6) are satisfied since the CoP stays between $-\ell_{\text {foot }}=-0.2$ and 0 and the friction stays between the limits, since $-\mu_{s} \leq \frac{F x}{F y} \leq \mu_{s}$, where $\mu_{s}$ equals 0.7 .

We compared our methodology with a classic nonswitching approach where we apply Eq. (21) only once after the impact and the reference values of the initially chosen primitive are tracked for the whole motion. The cost $I$ using this approach was 52.6697 , much higher than what the trajectory switching offers. 

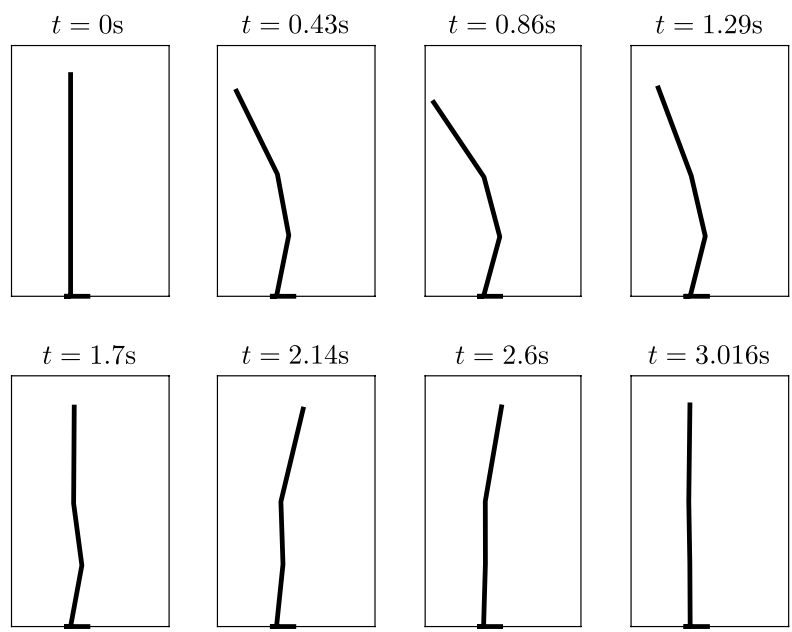

Fig. 4. Snapshots of the resulting balancing motion.

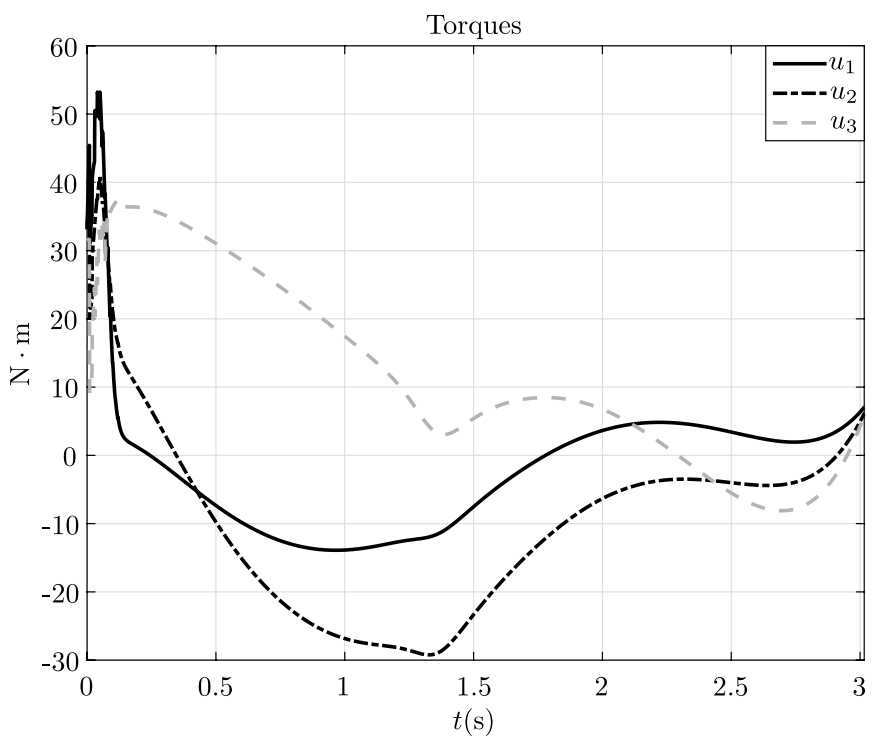

Fig. 5. Torques of the resulting balancing motion.

The sequence of best fitting Motion Primitives is presented in Fig. 7. As is shown, at $0.13 \mathrm{~s}$ the switching is shut off since the CLF constraint violation is below the assumed threshold of $\delta_{\text {thr }}=0.001$. Before that, it is shown that different Motion Primitives were chosen and tracked for either shorter or longer time.

Finally, in Fig. 8 we provide the final posture of the robot after an impact force of $\boldsymbol{F}_{\text {push }}=\left[\begin{array}{ll}60 & 25\end{array}\right]^{T}$ with an application point of 0.7 . As is shown, the trajectory 


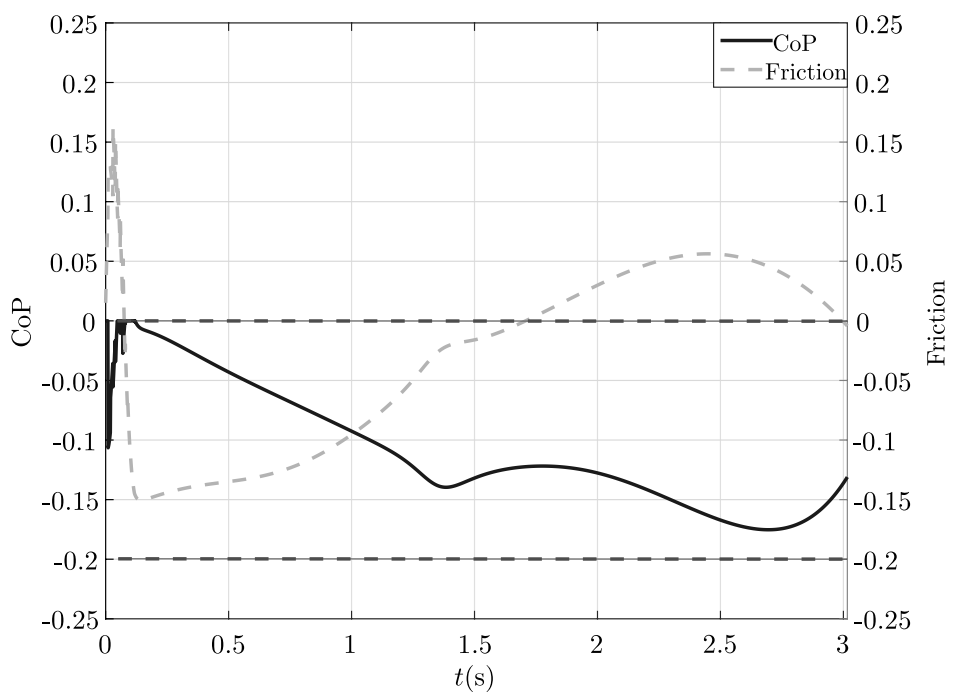

Fig. 6. CoP and friction cone associated with the resulting balancing motion. The dashed horizontal lines mark the limits for the CoP.

switching methodology allows for balancing while the classic approach of following only one primitive chosen at the beginning is not able to bring the robot in the rest posture in the allotted time. Regarding the cost $I$ of the motion, it is 14.8088 for the switching methodology and 122.41 for the classic approach mainly due to the fact

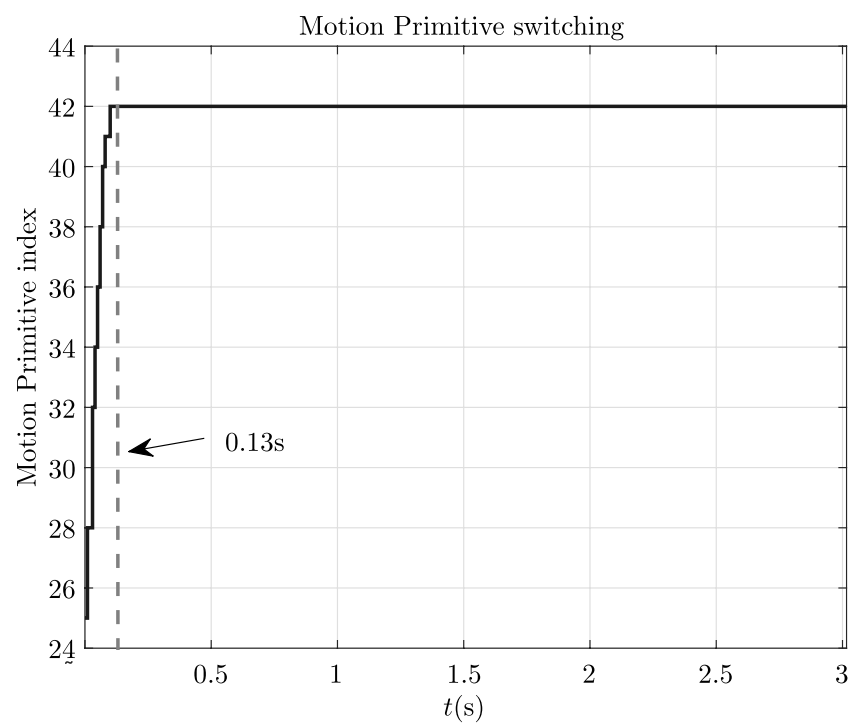

Fig. 7. Motion Primitives that choose the reference values to be passed to the QP. The dashed line indicates when the CLF constraint violation becomes smaller than the threshold $\delta_{\text {thr }}$ and the switching is shut off. 


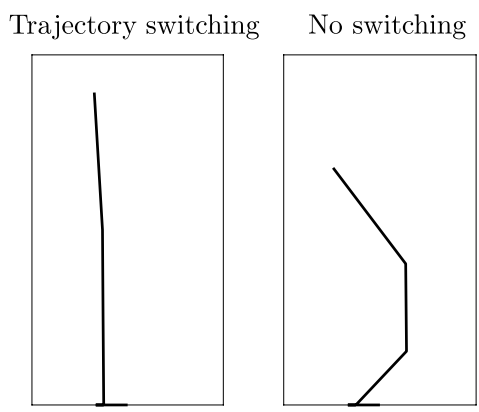

Fig. 8. Final posture of the robot after an impact force of $\boldsymbol{F}_{\text {push }}=\left[\begin{array}{ll}60 & 25\end{array}\right]^{T}$ with an application point of 0.7 following the switching approach (left) and not (right).

that the deviation between the desired and the actual terminal state of the robot is very large.

As is shown, our system is able to reject disturbances that were not presented during the offline optimization phase by employing the trajectory switching approach. This switching methodology allowed to generate balancing motions online which provide a lower cost in comparison to a nonswitching approach. Even for larger impact forces like in Fig. 8, our switching approach provides a balancing motion which is able to bring the robot closer to the upright posture in comparison to the nonswitching approach. At the same time, all the constraints imposed on the GRFs and torque limits are satisfied.

\subsection{Double impact experiment}

This experiment aims to show how the proposed methodology is able to balance the robot when it is further disturbed while not in the rest posture yet. For that, we start by applying an impact force $\boldsymbol{F}_{\text {push }}^{1}=\left[\begin{array}{ll}20 & 5\end{array}\right]^{T}$ with an application point of 0.68 . After $1 \mathrm{~s}$, while the robot has not reached the rest posture yet, we apply a second impact force $\boldsymbol{F}_{\text {push }}^{2}=\left[\begin{array}{ll}20 & 10\end{array}\right]^{T}$ with an application point of 0.65 . The resulting balancing motion has an overall cost of 4.457 .

By storing the database in terms of samples and applying such a switching approach we are able to endow these reactive characteristics to our approach. In order to detect the second impact we can take advantage of the fact that an impact will cause a considerable discontinuity in the joint velocities. If we have not chosen to switch again after the second impact, we would have to deal with a great divergence between the reference values provided by the primitive chosen at $t=0.13 \mathrm{~s}$ (when switching is shut off in Fig. 12) and the ones corresponding to the robot. Finally, if we choose one trajectory at $t=0 \mathrm{~s}$ and then another one at $t=1 \mathrm{~s}$ (after each impact) the cost is 6.9969, greater than our switching approach.

As is shown in Fig. 9, the robot is able to return to the rest posture while at the same time the torque limits (Fig. 10) and the CoP and friction constraints (Fig. 11) 

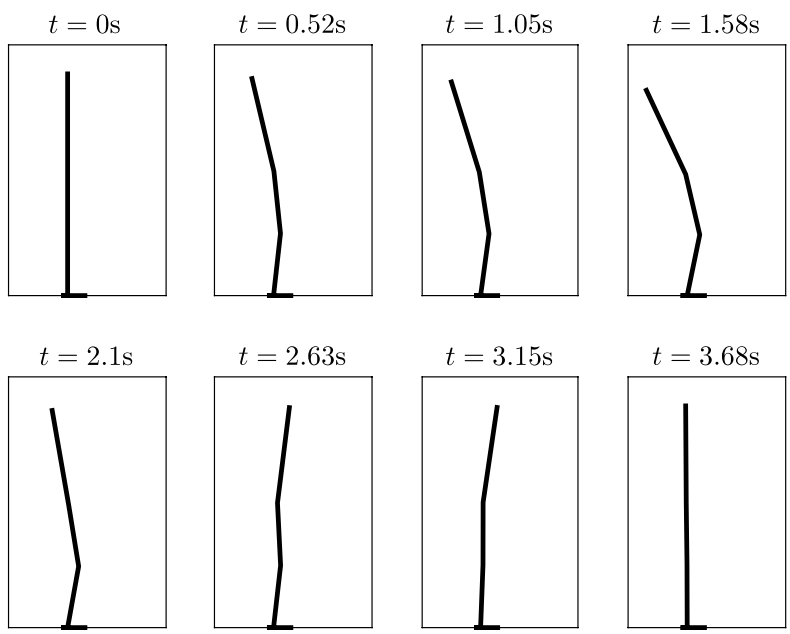

Fig. 9. Snapshots of the resulting balancing motion in the double impact experiment.

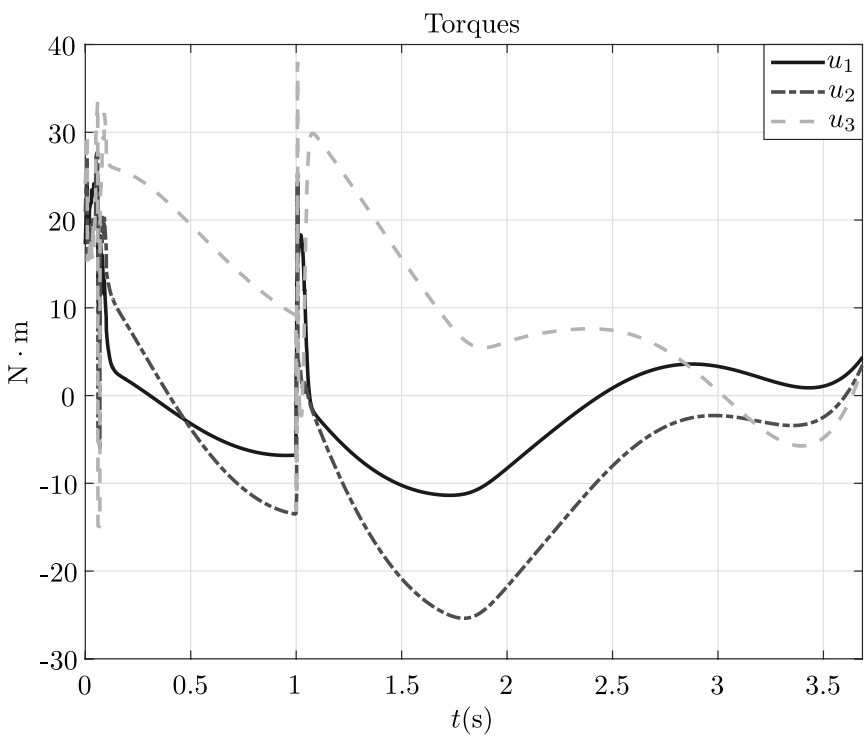

Fig. 10. Torques during the double impact experiment.

are all satisfied. Finally, Fig. 12 shows the Motion Primitive switching that takes place during the double impact experiment.

\subsection{Continuous push}

In this experiment we demonstrate that our approach can also deal with continuous pushes. For this purpose we apply for the first $400 \mathrm{~ms}$ a force $F_{\text {push }}=\left[\begin{array}{ll}15 & 0\end{array}\right]$ with an 


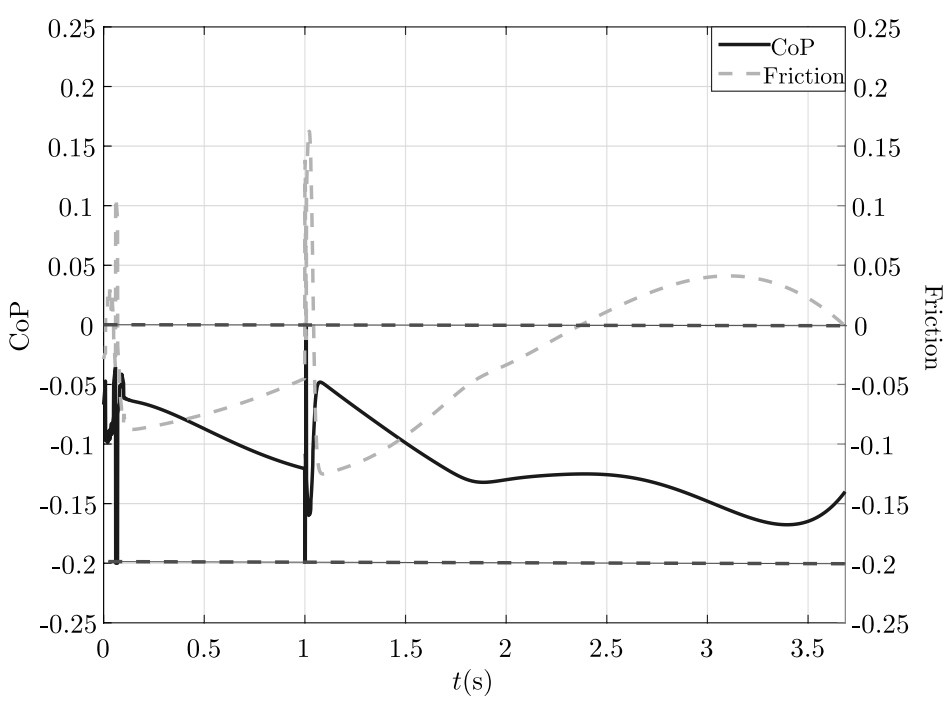

Fig. 11. CoP and friction cone associated with the double impact experiment. The dashed horizontal lines mark the limits for the CoP.

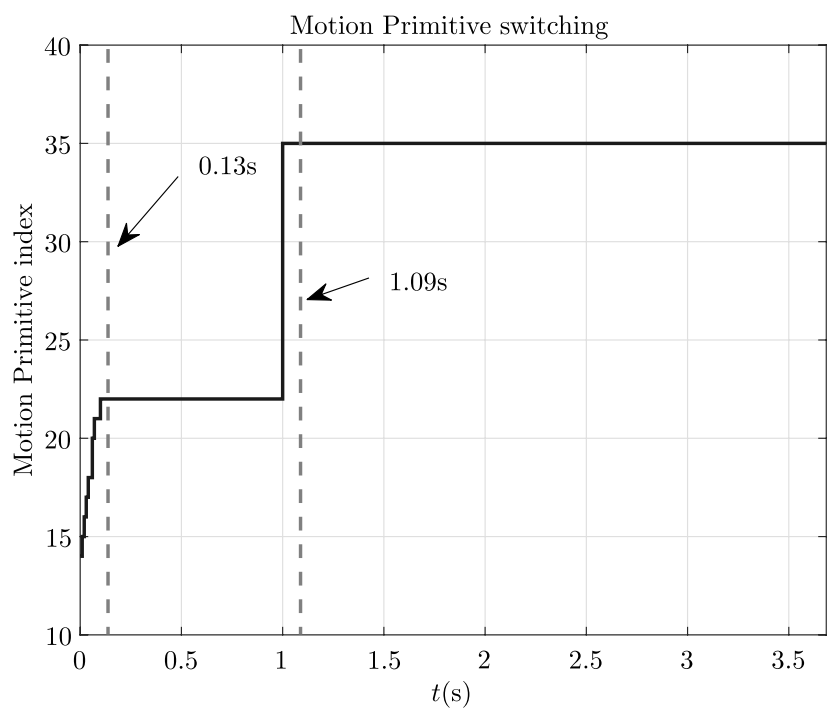

Fig. 12. Motion Primitives that choose the reference values to be passed to the QP for the double impact experiment. The dashed lines indicate when the CLF constraint violation becomes smaller than the threshold $\delta_{\text {thr }}$ and the switching is shut off.

application point of 1 , i.e., at the tip of the torso. What is interesting in this experiment is that as we can see in Fig. 13 the robot is able to return to the upright posture when applying a continuous push even though the database of primitives is generated for instantaneous impact forces. At the same time the constraints on the 

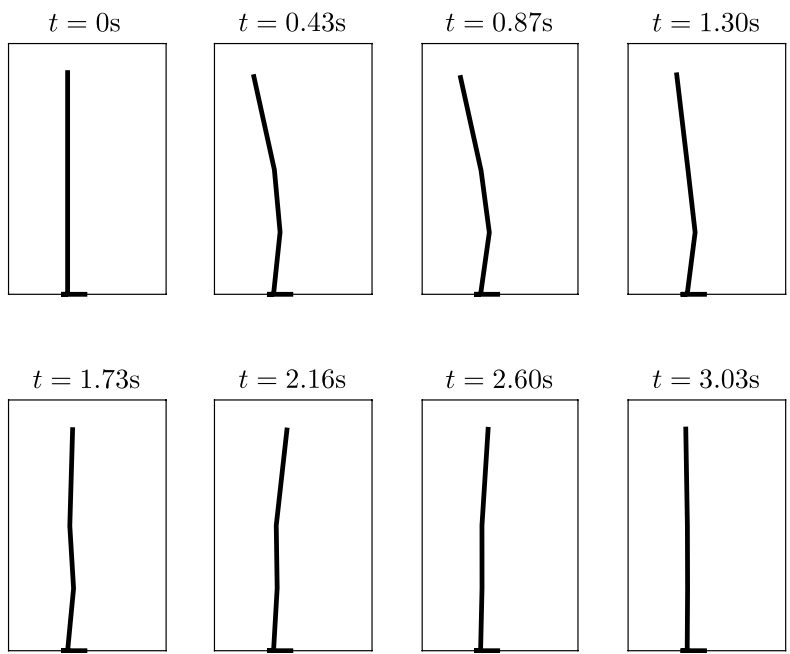

Fig. 13. Snapshots of the resulting balancing motion in the continuous push experiment.

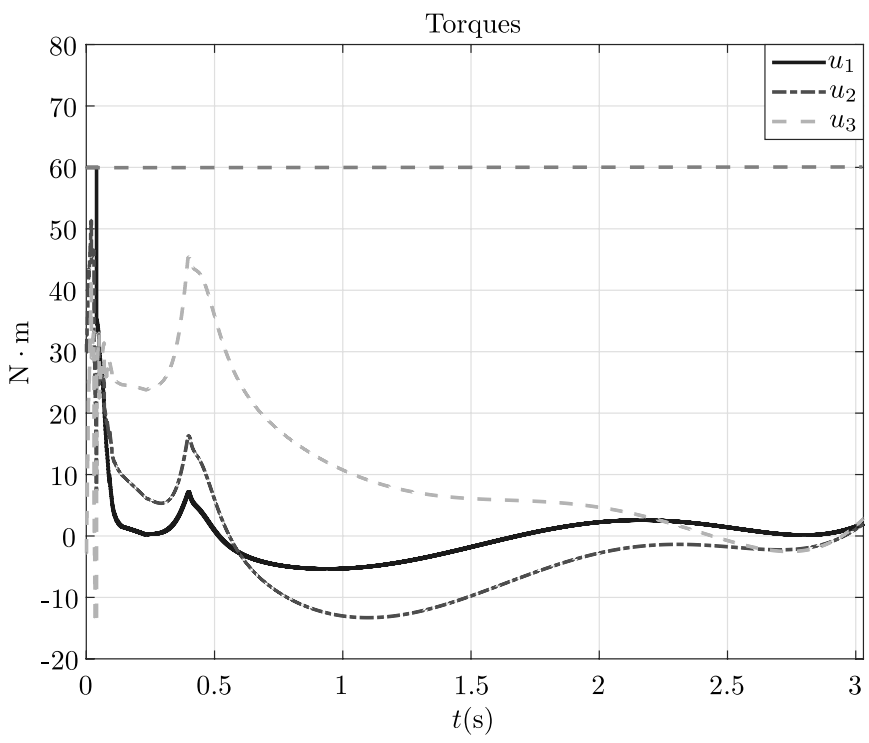

Fig. 14. Torques during the continuous push experiment.

input torques (Fig. 14) and GRFs (Fig. 15) are satisfied. This is due to the fact that we have stored each primitive in samples and we can switch between samples of different trajectories at each time step, enhancing in that way the capabilities of the database and our approach. In addition, as seen in Fig. 16 even though the switching 


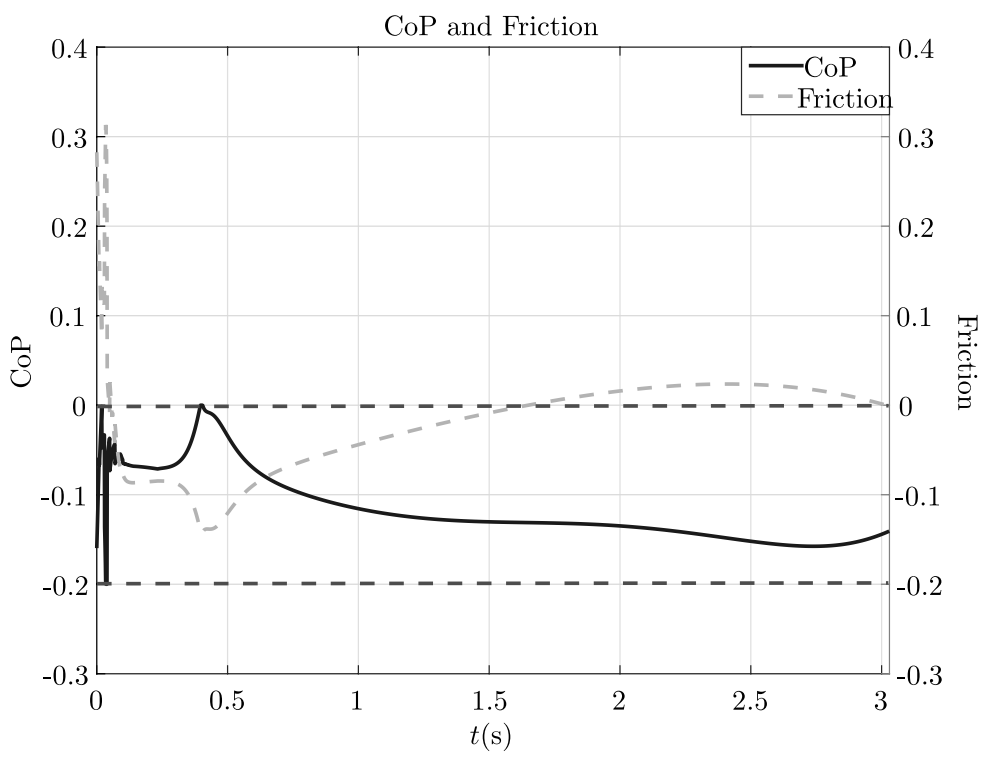

Fig. 15. CoP and friction cone associated with the continuous push experiment. The dashed horizontal lines mark the limits for the CoP.

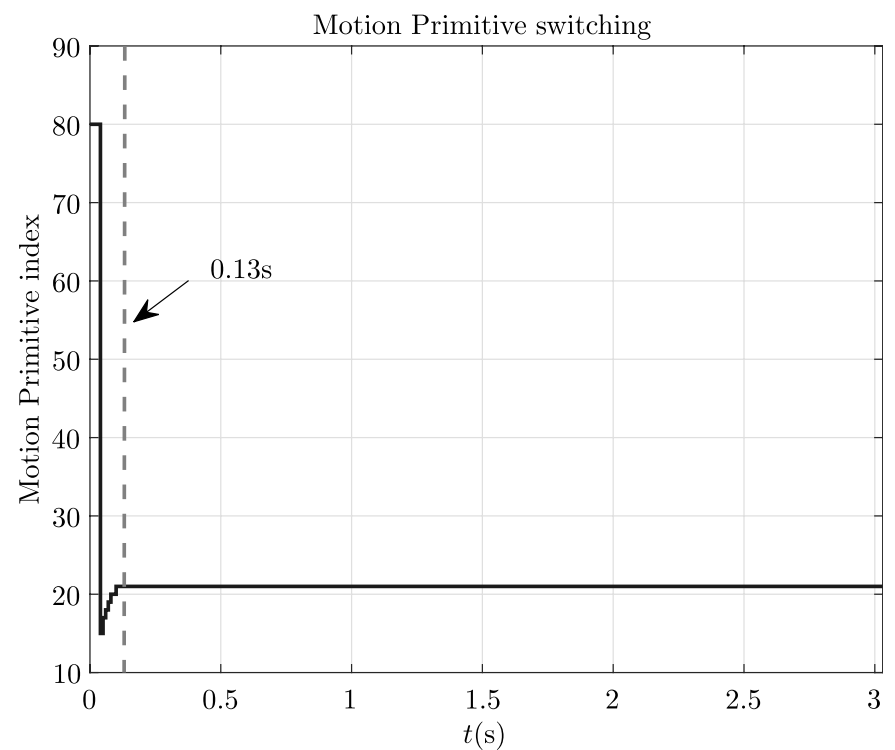

Fig. 16. Motion Primitives that choose the reference values to be passed to the QP for the continuous push experiment. The dashed line indicates when the CLF constraint violation becomes smaller than the threshold $\delta_{\text {thr }}$ and the switching is shut off. 


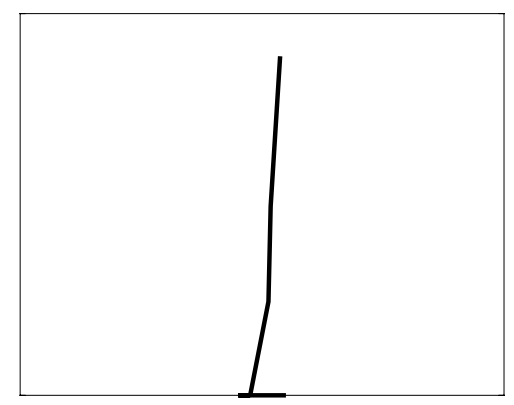

Fig. 17. Final posture of the robot that corresponds to the continuous push experiment when a nonswitching approach is used.

is turned off after $130 \mathrm{~ms}$, for the remaining $270 \mathrm{~ms}$ the robot is still able to withstand the push, due to the fact that after this point the motion dictated by the push and the one of the selected primitive are in good accordance with each other. Finally, if we discard the switching and try to balance the robot only with one trajectory chosen at the beginning of the motion, we are not able to bring the robot back in the upright posture (see Fig. 17).

\subsection{Model uncertainties - noisy measurements}

This experiment is intended to demonstrate the capabilities of our approach to handle model uncertainties and noisy measurements. For that purpose we assume that the robot model differs from the one we used in our offline optimization phase. More precisely, we assume that the inertia of the torso is $5 \%$ more, i.e., instead of 1.33 it is 1.3965 and the inertia of the femur is $2 \%$ less, i.e., instead of 0.94 it is now 0.9212 (compare also with Table 1). At the same time we assume that the information from the joint velocities is noisy and for this purpose we add Gaussian white noise with a variance of $\sigma^{2}=0.01$. We use the same disturbance scenario as in experiment 6.2 , i.e., $\boldsymbol{F}_{\text {push }}=\left[\begin{array}{ll}40 & 25\end{array}\right]^{T}$ with an application point of 0.7 .

In Fig. 18 we see that the robot is able to return to the upright posture by applying the switching methodology. In Figs. 19 and 21 we see that the torques stay inside the assumed bounds and that the CoP and friction cone constraints are both satisfied. The torques and the GRFs, however, have spikes which is expected, since we have severely changed the parameters of our model and have included noise. The chattering phase of the torques and the constraints on the GRFs between $0.5 \mathrm{~s}$ and $1 \mathrm{~s}$ corresponds to high noise in the joint velocities. For that reason, we also plot the joint velocities during the uncertainty experiment in Fig. 20. Finally, the Motion Primitive switching that takes place during this experiment is shown in Fig. 22.

In order to get an estimate of the cost, we ran this experiment 10 times, five times with the switching approach and five with the nonswitching. The cost of each trial is shown in Table 4, where the average cost for the switching approach is 8.2476 and 14.938 for the nonswitching. The figures correspond to the third trial which has 

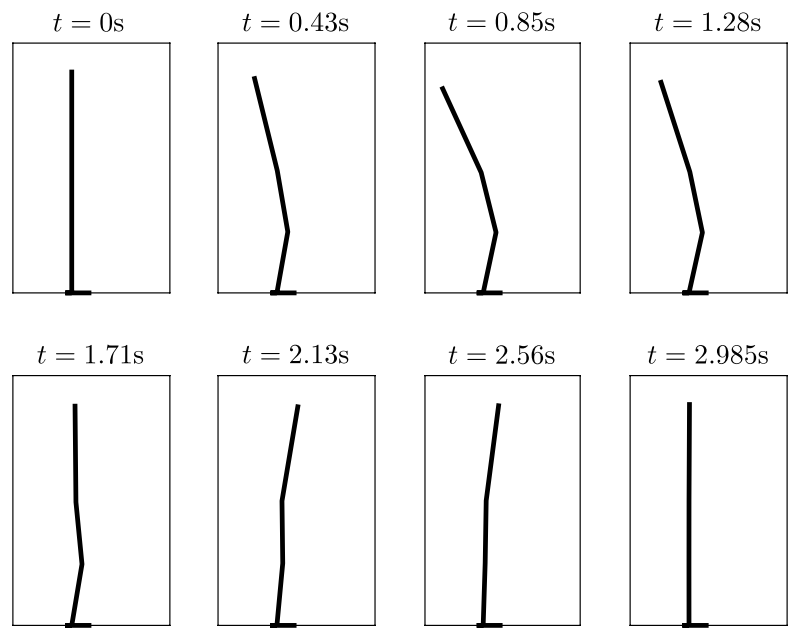

Fig. 18. Snapshots of the resulting balancing motion in the model uncertainty experiment.

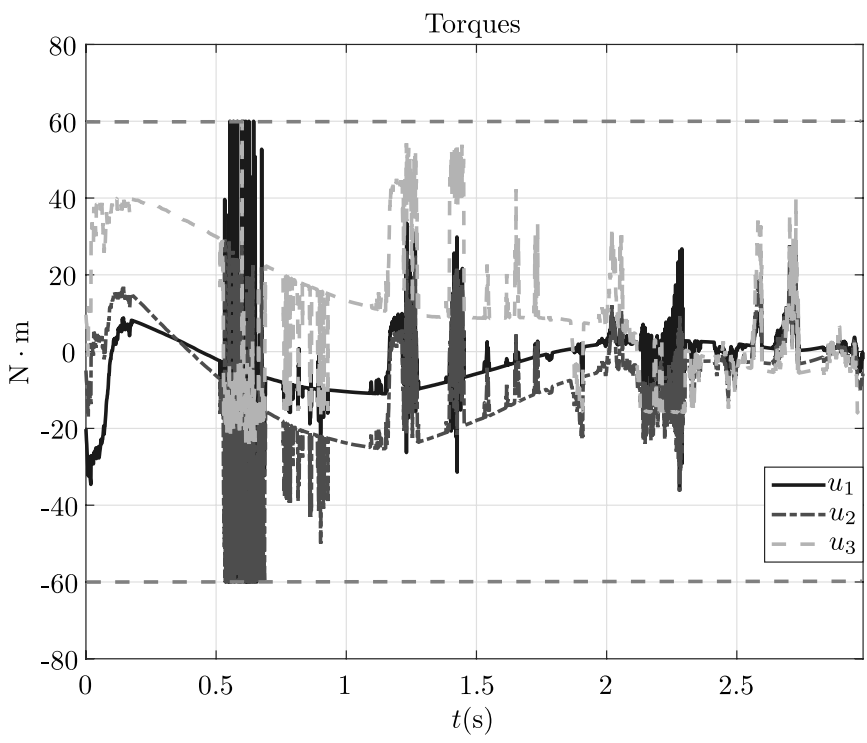

Fig. 19. Torques during the model uncertainty experiment.

a cost of 7.7048, which is close to the one during the experiment without any uncertainties and noise. As is shown, the parameters in Table 3 provide satisfactory results in case of model and measurement uncertainties. A positive aspect of the values adopted in our $\boldsymbol{L}$ matrix in the selection process is that we rely more on the joint positions in comparison to the joint velocities, since in a practical application the joint velocities are more noisy than the positions. 


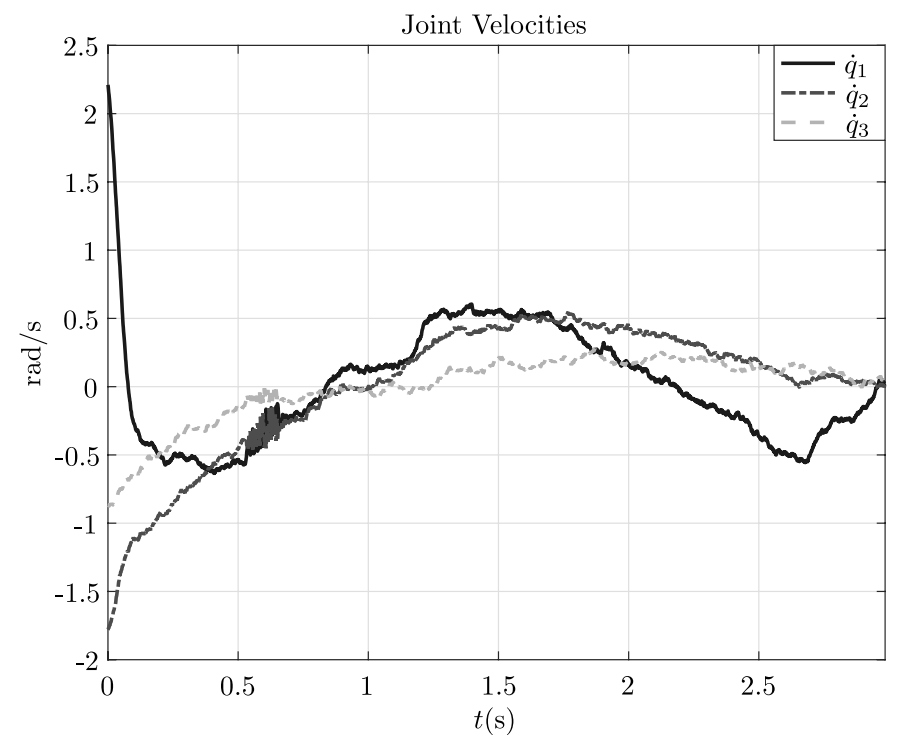

Fig. 20. Joint velocities during the model uncertainty experiment.

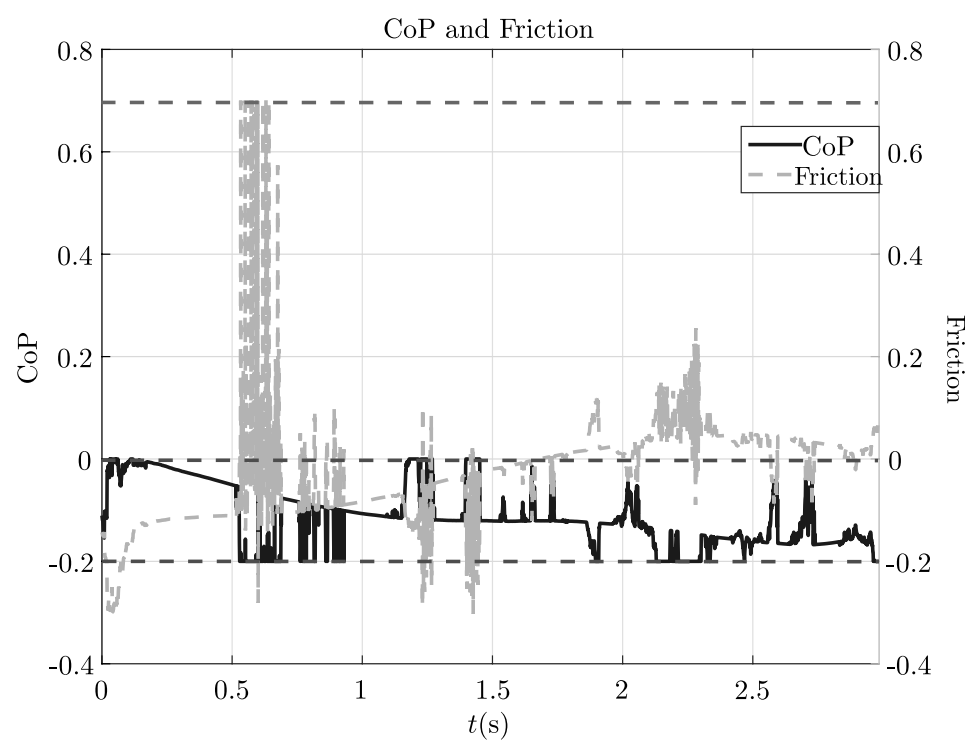

Fig. 21. CoP and friction cone associated with the model uncertainty experiment. The dashed horizontal lines mark the limits for the CoP and the friction.

\subsection{Overall evaluation}

This experiment is intended to demonstrate how the switching methodology can enhance the performance of the system in comparison to the classic approach of 


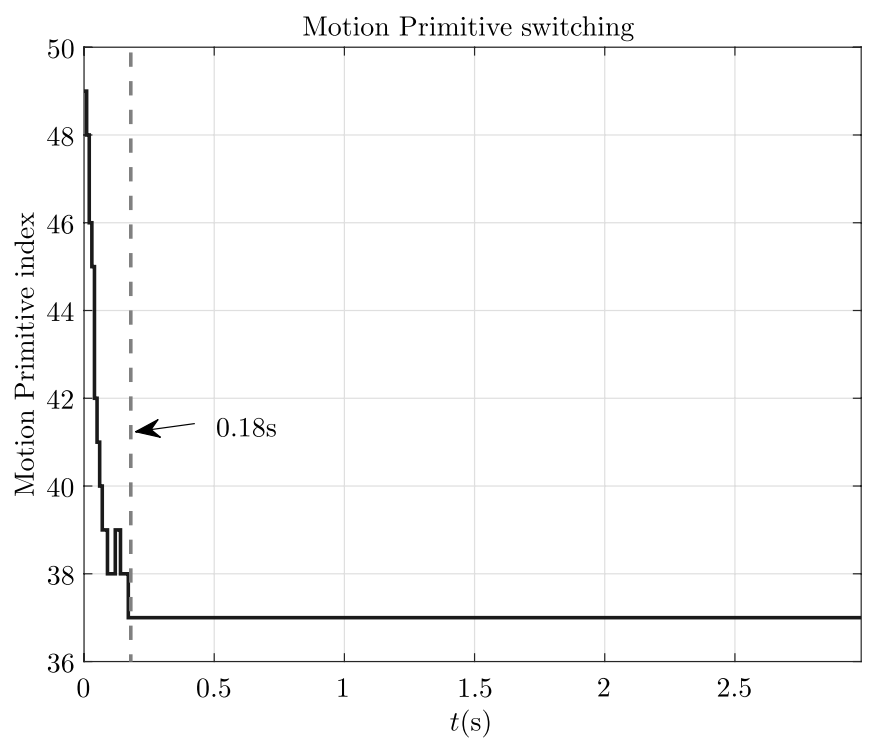

Fig. 22. Motion Primitives that choose the reference values to be passed to the QP for the model uncertainty experiment. The dashed line indicates when the CLF constraint violation becomes smaller than the threshold $\delta_{\text {thr }}$ and the switching is shut off.

Table 4. Cost of different trials of the model uncertainty and measurement noise experiment.

\begin{tabular}{lrrrrrr}
\hline Trial & \multicolumn{1}{c}{1} & \multicolumn{1}{c}{2} & \multicolumn{1}{c}{3} & \multicolumn{1}{c}{4} & \multicolumn{1}{c}{5} & Average \\
\hline Nonswitching & 18.1472 & 12.2432 & 16.3817 & 14.9073 & 13.0104 & 14.938 \\
Switching & 7.2763 & 9.2084 & 7.7048 & 8.4291 & 8.2057 & 8.2476 \\
\hline
\end{tabular}

choosing a primitive only at the beginning of the motion in order to provide the reference motion. The comparison is performed in terms of cost $I$ (Eq. (4)). The chosen grid consists of impact forces with a tangent and normal component and an application point of 0.7. As shown in Fig. 23, the cost difference is greater in favor of the trajectory switching methodology, showing that in terms of efficiency there is a direct advantage in performing trajectory switching rather than committing to only one trajectory chosen at the beginning of the motion. The advantage is more evident as the normal component of the impact force $F_{\text {push }}^{x}$ increases since the difference between the post-impact state of the robot and the state provided by the initially chosen Motion Primitive sample increases. As a consequence, the nonswitching policy will need time to converge to the optimal values provided by the chosen Motion Primitive. In contrast, the switching policy has the opportunity to choose a Motion Primitive sample $P_{[i, k]}^{*}$ that has a smaller Euclidean distance to the current state of the robot and converge to the optimal values of the $i$ th-Motion Primitive faster. 


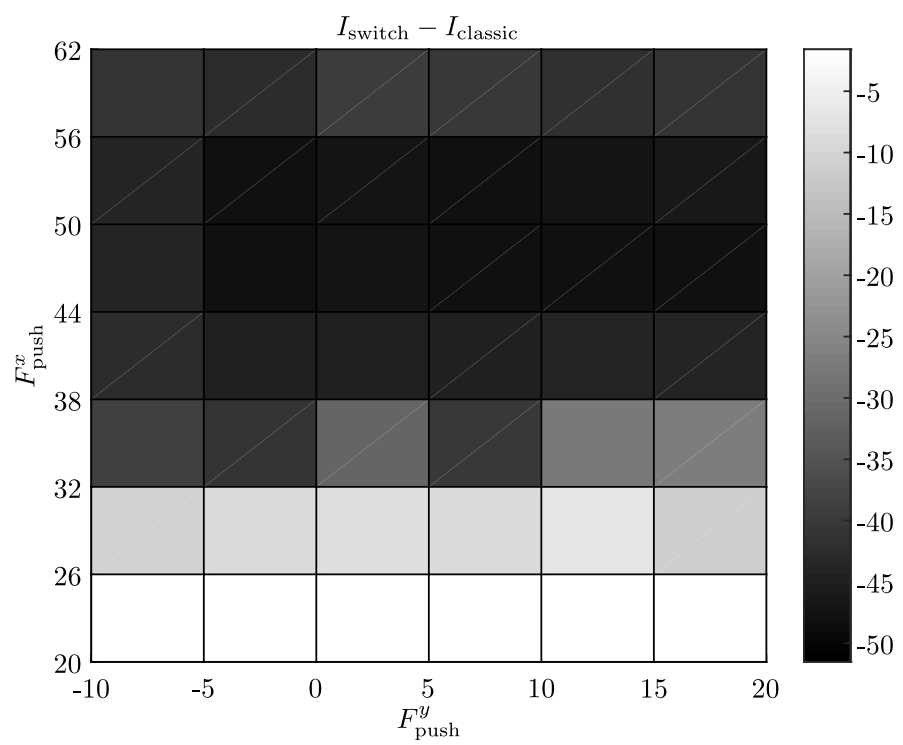

Fig. 23. An overall evaluation of the cost of the proposed methodology $I_{\text {switch }}$ compared to the cost of the classic nonswitching approach $I_{\text {classic }}$ where we use the same primitive during the whole motion. As shown, the switching methodology can provide more efficient balancing motions for a wide range of impact forces $F_{\text {push }}$.

\section{Discussion and Conclusions}

This paper introduced a balancing methodology based on Motion Primitives samples. At first, different balancing motions are calculated. Each motion brings the robot back to the rest posture after a different impact force is applied. As a further step, the trajectories, accelerations, torques and GRFs associated with each balancing motion are sampled and form a database of Motion Primitive samples. When the robot is disturbed with an unknown impact force, a Motion Primitive switching algorithm takes place. For every $T_{n}$ seconds the database is searched for samples with the current time stamp $k T_{n}$ and the one closest to the current state of the robot - in terms of a Euclidean distance metric - is chosen. In such a way, for the next $T_{n}$ seconds reference trajectories, torques and GRFs to be tracked are generated by the Motion Primitive to which the last selected sample belongs. In order to ensure that the tracking will not result in a motion that violates the modeling assumptions, we solve a Quadratic Program online every $T_{s} \leq T_{n}$ seconds where all these modeling assumptions are treated as constraints. The cost function penalizes the deviation from the input torques, GRFs and accelerations dictated by the current Motion Primitive. In addition, a Control Lyapunov Constraint is introduced that ensures exponential convergence to the desired positions and velocities at a pre-defined rate $\varepsilon$. The violation of the Control Lyapunov Constraint is penalized in the cost function as well and when it becomes lower than a user defined threshold the switching is discarded and reference values to track are generated by the lastly used primitive. 
The results show that this methodology can provide solutions for different impact scenarios in a robust fashion with a smaller cost than if we chose to track a sole Motion Primitive right after the impact. Additionally, it can provide balancing motions for impact forces for which a solution cannot be found by offline optimization.

For the future, we propose to investigate the connections between the weighting matrix $\boldsymbol{L}$ of the selection methodology and the time $T_{n}$ with the cost of the overall motion, such that we can derive probabilistic guarantees that the chosen samples will produce a balancing motion with minimum cost. It is also interesting to be able to find the minimum number of primitives that are necessary to balance the robot for a wide range of impact forces. To this end, we move away from optimality and focus on the more fundamental problem of covering a big state space with a minimum number of controllers/trajectories, where interesting works — regarding an incremental building of the database — can be found in the Machine Learning bibliography. ${ }^{41-43}$ Finally, we would like to test our methodology on a real robot, where further constraints on the torque derivative should be taken into account to ensure a smooth and non-jerky motion.

\section{Acknowledgments}

The research leading to these results has received funding partly from the European Research Council under the European Unions Seventh Framework Program (FP/ 2007-2013)/ERC Grant Agreement No. [267877] and partly from the Technical University of Munich — Institute for Advanced Study (www.tum-ias.de), funded by the German Excellence Initiative.

\section{References}

1. S. Kajita, F. Kanehiro, K. Kaneko, K. Yokoi and H. Hirukawa, The 3D linear inverted pendulum mode: A simple modeling for a biped walking pattern generation, IEEE/RSJ Int. Conf. Intelligent Robots and Systems, Vol. 1 (2001), pp. 239-246.

2. S.-H. Lee and A. Goswami, Reaction mass pendulum (RMP): An explicit model for centroidal angular momentum of humanoid robots, IEEE Int. Conf. Robotics and Automation (2007), pp. 4667-4672.

3. C. G. Atkeson and B. Stephens, Multiple balance strategies from one optimization criterion, IEEE-RAS Int. Conf. Humanoid Robots (2007), pp. 57-64.

4. J. Pratt, J. Carff, S. Drakunov and A. Goswami, Capture point: A step toward humanoid push recovery, IEEE-RAS Int. Conf. Humanoid Robots (2006), pp. 200-207.

5. J. A. Castano, Z. Li, C. Zhou, N. Tsagarakis and D. Caldwell, Dynamic and reactive walking for humanoid robots based on foot placement control, Int. J. Hum. Robot. 13(2) (2016) 1550041.

6. Z. Aftab, T. Robert and P. B. Wieber, Ankle, hip and stepping strategies for humanoid balance recovery with a single model predictive control scheme, IEEE-RAS Int. Conf. Humanoid Robots (Humanoids) (2012), pp. 159-164.

7. B. J. Stephens and C. G. Atkeson, Push recovery by stepping for humanoid robots with force controlled joints, IEEE-RAS Int. Conf. Humanoid Robots (2010), pp. 52-59. 
8. A. Kuo, An optimal control model for analyzing human postural balance, IEEE Trans. Biomed. Engrg. 42(1) (1995) 87-101.

9. P. A. Parrilo, Structured Semidefinite Programs and Semialgebraic Geometry Methods in Robustness and Optimization, Ph.D. thesis (California Institute of Technology, 2000).

10. R. Tedrake, I. R. Manchester, M. Tobenkin and J. W. Roberts, LQR-trees: Feedback motion planning via sums-of-squares verification, Int. J. Robot. Res. 29(8) (2010) 1038-1052.

11. P. Sardain and G. Bessonnet, Forces acting on a biped robot. center of pressure-zero moment point, IEEE Trans. Syst., Man, Cybern. A, Syst. Hum. 34(5) (2004) 630-637.

12. C. Liu and C. Atkeson, Standing balance control using a trajectory library, IEEE Int. Conf. Intelligent Robots and Systems (2009), pp. 3031-3036.

13. B. Stephens and C. Atkeson, Dynamic balance force control for compliant humanoid robots, IEEE/RSJ Int. Conf. Intelligent Robots and Systems (2010), pp. 1248-1255.

14. K. Yamane and Y. Nakamura, Dynamics filter - Concept and implementation of online motion generator for human figures, IEEE Trans. Robot. Autom. 19(3) (2003) 421-432.

15. S. H. Hyon, J. G. Hale and G. Cheng, Full-body compliant human-humanoid interaction: Balancing in the presence of unknown external forces, IEEE Trans. Robot. 23(5) (2007) 884-898.

16. S.-H. Lee and A. Goswami, A momentum-based balance controller for humanoid robots on non-level and non-stationary ground, Auton. Robots 33(4) (2012) 399-414.

17. A. Herzog, N. Rotella, S. Mason, F. Grimminger, S. Schaal and L. Righetti, Momentum control with hierarchical inverse dynamics on a torque-controlled humanoid, Auton. Robots 40(3) (2016) 473-491.

18. A. Herzog, L. Righetti, F. Grimminger, P. Pastor and S. Schaal, Balancing experiments on a torque-controlled humanoid with hierarchical inverse dynamics, 2014 IEEE/RSJ Int. Conf. Intelligent Robots and Systems (2014), pp. 981-988.

19. A. Escande, N. Mansard and P.-B. Wieber, Hierarchical quadratic programming: Fast online humanoid-robot motion generation, Int. J. Robot. Res. 33(7) (2014) 1006-1028.

20. S. Kuindersma, F. Permenter and R. Tedrake, An efficiently solvable quadratic program for stabilizing dynamic locomotion, IEEE Int. Conf. Robotics and Automation (ICRA) (2014), pp. 2589-2594.

21. J. Englsberger, C. Ott and A. Albu-Schäffer, Three-dimensional bipedal walking control using divergent component of motion, IEEE/RSJ Int. Conf. Intelligent Robots and Systems (2013), pp. 2600-2607.

22. M. A. Hopkins, A. Leonessa, B. Y. Lattimer and D. W. Hong, Optimization-based wholebody control of a series elastic humanoid robot, Int. J. Hum. Robot. 13(1) (2016) 1550034.

23. S. Apostolopoulos, M. Leibold and M. Buss, Online motion planning over uneven terrain with walking primitives and regression, IEEE Int. Conf. Robotics and Automation (ICRA) (2016), pp. 3799-3805.

24. A. D. Ames, K. Galloway, K. Sreenath and J. W. Grizzle, Rapidly exponentially stabilizing control Lyapunov functions and hybrid zero dynamics, IEEE Trans. Autom. Control 59(4) (2014) 876-891.

25. D. A. Levinson and T. R. Kane, AUTOLEV - A new approach to multibody dynamics, in Multibody Systems Handbook (Springer, Berlin, Heidelberg, 1990), pp. 81-102.

26. M. Vukobratović and B. Borovac, Zero-moment point thirty-five years of its life, Int. J. Hum. Robot. 1(1) (2004) 157-173.

27. J. Denk and G. Schmidt, Synthesis of walking primitive databases for biped robots in 3D environments, IEEE Int. Conf. Robotics and Automation, 2003, Vol. 1 (2003), pp. 13431349 . 
28. K. Koch, K. Mombaur, O. Stasse and P. Soueres, Optimization-based exploitation of the ankle elasticity of HRP-2 for overstepping large obstacles, IEEE-RAS Int. Conf. Humanoid Robots (Humanoids) (2014), pp. 733-740.

29. J. Morimoto, G. Cheng, C. G. Atkeson and G. Zeglin, A simple reinforcement learning algorithm for biped walking, IEEE Int. Conf. Robotics and Automation, 2004, Vol. 3 (2004), pp. 3030-3035.

30. J. Lee and J. H. Oh, Biped walking pattern generation using reinforcement learning, Int. J. Hum. Robot. 6(1) (2009) 1-21.

31. J. Hwangbo, C. Gehring, H. Sommer, R. Siegwart and J. Buchli, Policy learning with an efficient black-box optimization algorithm, Int. J. Hum. Robot. 12(3) (2015) 1550029.

32. A. J. Ijspeert, J. Nakanishi, H. Hoffmann, P. Pastor and S. Schaal, Dynamical movement primitives: Learning attractor models for motor behaviors, Neural Comput. 25(2) (2013) 328-373.

33. P. Pastor, H. Hoffmann, T. Asfour and S. Schaal, Learning and generalization of motor skills by learning from demonstration, IEEE Int. Conf. Robotics and Automation (2009), pp. $763-768$.

34. A. J. Ijspeert, J. Nakanishi and S. Schaal, Learning attractor landscapes for learning motor primitives, in Proc. 15th Int. Conf. Neural Information Processing Systems, NIPS'O2 (MIT Press, Cambridge, MA, USA, 2002), pp. 1547-1554.

35. E. Theodorou, J. Buchli and S. Schaal, A generalized path integral control approach to reinforcement learning, J. Mach. Learn. Res. 11 (2010) 3137-3181.

36. Z. Artstein, Stabilization with relaxed controls, Nonlinear Anal.: Theory, Methods Appl. 7(11) (1983) 1163-1173.

37. E. D. Sontag, A universal construction of Artstein's theorem on nonlinear stabilization, Syst. Control Lett. 13(2) (1989) 117-123.

38. R. Kelly, V. Santibanez and A. Loria, Computed-torque control and computed-torque + control, in Control of Robot Manipulators in Joint Space (Springer, London, 2005), pp. $227-241$.

39. A. D. Ames and M. Powell, Towards the Unification of Locomotion and Manipulation Through Control Lyapunov Functions and Quadratic Programs, Springer's Lecture Notes in Control and Information Science (Springer, 2013), pp. 219-240.

40. H. Ferreau, C. Kirches, A. Potschka, H. Bock and M. Diehl, qpOASES: A parametric active-set algorithm for quadratic programming, Math. Program. Comput. 6(4) (2014) $327-363$.

41. F. Meier, E. Theodorou and S. Schaal, Movement segmentation and recognition for imitation learning, in Proc. Fifteenth Int. Conf. Artificial Intelligence and Statistics (AISTATS-12), eds. N. D. Lawrence and M. A. Girolami, Vol. 22 (2012), pp. 761-769.

42. D. Kulić, C. Ott, D. Lee, J. Ishikawa and Y. Nakamura, Incremental learning of full body motion primitives and their sequencing through human motion observation, Int. J. Robot. Res. (2011), Doi: 10.1177/0278364911426178.

43. A. Lemme, R. F. Reinhart and J. J. Steil, Self-supervised bootstrapping of a movement primitive library from complex trajectories, 2014 IEEE-RAS Int. Conf. Humanoid Robots (2014), pp. 726-732. 

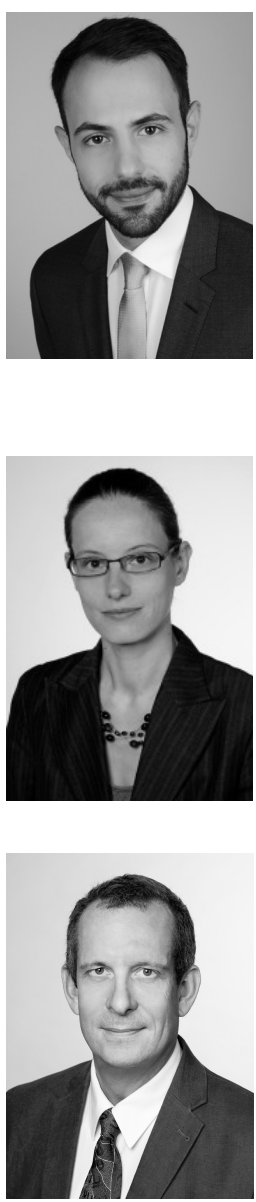

Sotiris Apostolopoulos received his Diploma degree in Electrical and Computer Engineering from the National Technical University of Athens, Greece, in 2012. He is currently employed as a Research Associate at the Chair of Automatic Control Engineering of the Technical University of Munich, Germany while pursuing the doctoral degree. His research interests include robotics, motion databases for robotic systems, humanoid locomotion and balancing.

Marion Leibold received her Diploma degree in Applied Mathematics and her Ph.D. degree in Electrical Engineering from the Technical University of Munich, Germany, in 2002 and 2007, respectively. She is currently a Lecturer in the Chair of Automatic Control Engineering of the Technical University of Munich. Her research interests include hybrid dynamical systems, optimal control and legged robots.

Martin Buss received the Diploma Engineering degree in Electrical Engineering from the Technical University Darmstadt, Darmstadt, Germany, in 1990, the Doctor of Engineering degree in Electrical Engineering from the University of Tokyo, Tokyo, Japan, in 1994, and the Habilitation degree from the Technical University of Munich, Munich, Germany, in 2000. In 1988, he was a Research Student for one year with the Science University of Tokyo. From 1994-1995, he was a Postdoctoral Researcher with the Department of Systems Engineering, Australian National University, Canberra, Australia. From 1995-2000, he was a Senior Research Assistant and a Lecturer with the Chair of Automatic Control Engineering, Department of Electrical Engineering and Information Technology, Technical University of Munich. From 2000-2003, he was a Full Professor, the Head of the Control Systems Group, and the Deputy Director of the Institute of Energy and Automation Technology, Faculty IV, Electrical Engineering and Computer Science, Technical University Berlin, Berlin, Germany. Since 2003, he has been a Full Professor (Chair) with the Chair of Automatic Control Engineering, Faculty of Electrical Engineering and Information Technology, Technical University of Munich, where he has been with the Medical Faculty since 2008. Since 2006, he has also been the Coordinator of the Deutsche Forschungsgemeinschaft Excellence Research Cluster Cognition for Technical Systems with CoTeSys. His research interests include automatic control, mechatronics, multimodal human system interfaces, optimization, nonlinear and hybrid discretecontinuous systems. 